\title{
Acta Neurochirurgica \\ Craniectomy size for subdural haematomas and the impact on brain shift and outcomes \\ --Manuscript Draft--
}

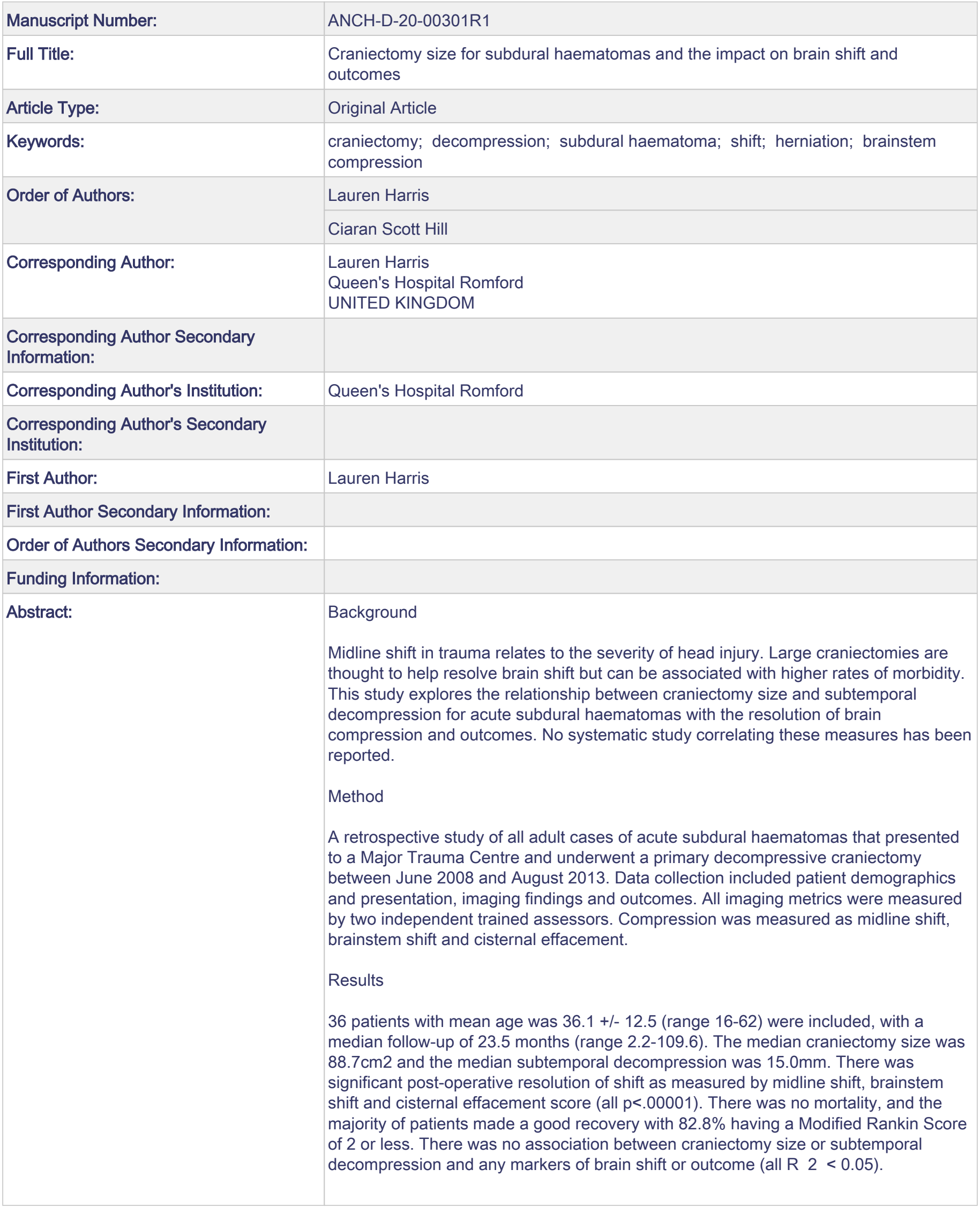




\section{Conclusions}

This study suggests that there is no clear relationship between craniectomy size or extent of subtemporal decompression and resolution of brain shift or outcome. Further studies are needed to assess the relative efficacy of large craniectomies and the role of subtemporal decompression. 
$4^{\text {th }}$ March 2020

Dear T. Mathiesen,

Editor of Acta Neurochirurgica

We would be grateful if you would consider our manuscript "Craniectomy size for subdural haematomas and the impact on brain shift and outcomes" for publication in your journal. We believe that our paper will be a valuable addition to the literature and will be highly cited, being the first study that explores the relationship between craniectomy size and degree of subtemporal decompression and resolution of compression and outcomes.

Background: Head injury is a leading cause of morbidity and mortality in people aged between one and forty years. Primary decompressive craniectomy attempts to reduce global ICP, focal compression and subsequent herniation with the aim to increase survival and reduce morbidity. Compression is assessed by radiological midline shift at the level of the lateral ventricles and by brainstem compression inferred as the presence or absence of cisternal effacement. Although it has not been formally demonstrated it is intuitive that resolution of compression would occur with decompression, and correlate with improved outcome.

Aims: Our primary aims were (i) does the size of the craniectomy relate to the degree of midline shift, brainstem shift, or cisternal effacement resolution? (ii) does the extent of subtemporal decompression relate to the degree of midline shift, brainstem shift, or cisternal effacement resolution? The secondary aim was to look at the longterm patient outcomes.

Cohort: 36 patients who underwent a primary decompressive craniectomy for an acute subdural haematoma between June 2008 and August 2013 and fulfilled our inclusion criteria were included, and followed-up for 23.5months.

Our findings: The median craniectomy size was $88.7 \mathrm{~cm}^{3}$ and the median subtemporal decompression was $15.0 \mathrm{~mm}$. There was significant post-operative resolution of shift as measured by midline shift, brainstem shift and cisternal effacement score (all $p<.00001$ ). There was no mortality, and the majority of patients made a good recovery with $82.8 \%$ having a Modified Rankin Score of 2 or less. There was no association between craniectomy size or subtemporal decompression and any markers of brain shift or outcome (all $R^{2}<0.05$ ).

Impact: This study suggests that there is no clear relationship between craniectomy size or extent of subtemporal decompression and resolution of brain shift or outcome. Despite our manuscript having certain limitations, our publication is likely to be highly sited and to be used by health care institutions as part of their craniectomy guidelines. Further studies are needed to assess the relative efficacy of large craniectomies and the role of subtemporal decompression

Yours sincerely, Lauren Harris 
<smiles>C1=CC2CCCCC2C1</smiles> 


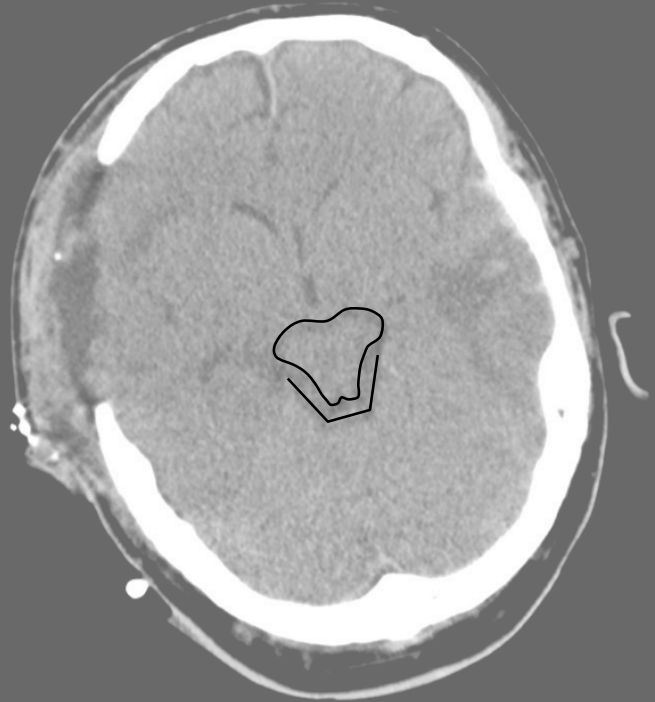




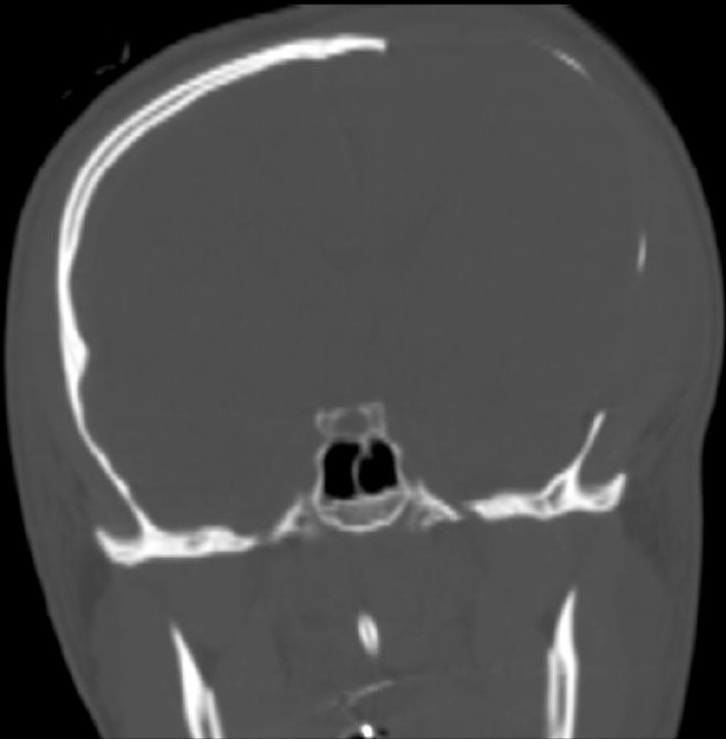




\section{Checklist for authors $27^{\text {th }}$ October 2016}

The journal is interested in original papers which are novel and carry important scientific information in the submitted journal field.

Format is important for the journal. We believe that format benefits the quality of science and we are convinced that a good format will enable reviewers and readers to grasp the intellectual contents better. The instructions for authors must be followed.

Duplicate submissions: Further advice regarding "what is a duplicate submission" can be found on the following website. The website discusses in detail plagiarism and ethical writing from the Office of Research Integrity.

\section{http://ori.dhhs.gov/education/products/plagiarism/}

\section{Applicability of the following reporting guidelines:}

Checklists have been developed for a number of study designs, including randomized controlled trials (CONSORT), systematic reviews (PRISMA), observational studies (STROBE), diagnostic accuracy studies (STARD) and qualitative studies (COREQ, RATS).

We recommend authors refer to the EQUATOR Network website (http://www.equator-network.org) for further information on the available reporting guidelines for health research, and the MIBBI Portal for prescriptive checklists for reporting biological and biomedical research where applicable. Authors are requested to make use of these when drafting their manuscript and peer reviewers will also be asked to refer to these checklists when evaluating these studies.

Please follow the checklist and check the following points:

$\underline{\mathbf{y}}$ The Title should be objective, short and concise, include a keyword in the title. Avoid using the words: Novel, Unique, First

$\mathbf{y} \quad$ Abbreviations and acronyms: Please include a list and place after the Title Page. Please use only accepted acronyms and abbreviations and avoid introducing new unless necessary for improved comprehension or "readability".

$\underline{\mathbf{y}}$ The abstract should be divided as follows:

Background - Why was the study carried out/Why is this case report so special

Methods - What did the research group do?

Results - What did the research group find?

Conclusions- What might this research mean?

Number the page-the abstract page will be page\#2 


\section{Introduction:}

If possible try to keep the introduction short, while mentioning the following points:

y Introduce the topic and give an objective review of the background

y Identify an explicit gap of knowledge; the reason why the study is needed

y Please write down the gap of knowledge briefly:

No study correlates the relationship between craniectomy size and degree of subtemporal decompression with the resolution of compression and outcomes.

$\mathbf{y}$ Define an aim based on the gap of knowledge

y Please write down the aim briefly:

The primary aims were (i) does the size of the craniectomy relate to the degree of midline shift, brainstem shift, or cisternal effacement resolution? (ii) does the extent of subtemporal decompression relate to the degree of midline shift, brainstem shift, or cisternal effacement resolution? The secondary aim was to look at the long-term patient outcomes.

$\mathbf{y}$ The material and methods-section should contain information that allows reproduction of you study (in principle) and allows tracing of your results.

y This is the section where statistics should be placed.

$\underline{\mathbf{V}}$ Do not forget that for chemicals or equipment the name and location should be given in parentheses.

y Describe how the participants /cells in your study were selected and excluded.

Do not describe standard methods in detail - use references

\section{Results:}

The best way to write a good result section is to follow the four golden rules:

Keep it relevant

Let the text tell the story

Let the tables give the evidence 
Let the figures/Video highlight and illustrate the novel information described in the paper

$\mathbf{y}$ Results of all analyses should be included, and all results that are noted in the Discussion need to be shown here

\section{Discussion:}

Please discuss in your own words the following:

y State and discuss the principal finding. This finding should be a result of the aim.

$\mathbf{y}$ Discuss the strengths and weakness of the study comparing it with other studies with different results. Are the findings reliable and generalizable?

$\mathbf{y}$ Discuss the possible impact of the study and implications

$y \quad$ Discuss the unanswered questions and the possibility of future research with explicit recommendations of what is a "next step".

\section{Conclusion:}

y This should be a brief summary of your principal finding and what it means.

y Please state the conclusion briefly:

This study suggests that there is no clear relationship between craniectomy size or extent of subtemporal decompression and resolution of brain shift or outcome.

\section{New Springer Guidelines:}

Please place the following headings prior to the reference section.

Funding: XXX provided financial support in the form of XX funding.

The sponsor had no role in the design or conduct of this research.

If no funding was received, please write:

Funding: No funding was received for this research. 
Conflict of Interest: All authors certify that they have no affiliations with or involvement in any organization or entity with any financial interest (such as honoraria; educational grants; participation in speakers' bureaus; membership, employment, consultancies, stock ownership, or other equity interest; and expert testimony or patent-licensing arrangements), or non-financial interest (such as personal or professional relationships, affiliations, knowledge or beliefs) in the subject matter or materials discussed in this manuscript.

Ethical approval: All procedures performed in studies involving human participants were in accordance with the ethical standards of the institutional and/or national research committee and with the 1964 Helsinki declaration and its later amendments or comparable ethical standards.

For retrospective studies, please add the following sentence:

"For this type of study formal consent is not required."

Informed consent: Informed consent was obtained from all individual participants included in the study. If identifying information about participants is available in the article, the following statement should be included:

"Additional informed consent was obtained from all individual participants for whom identifying information is included in this article."

\section{Animal Experiments:}

Ethical approval: "All applicable international, national, and/or institutional guidelines for the care and use of animals were followed."

If applicable (where such a committee exists): "All procedures performed in studies involving animals were in accordance with the ethical standards of the institution or practice at which the studies were conducted."

If articles do not contain studies with human participants or animals by any of the authors, please select one of the following statements:

"This article does not contain any studies with human participants performed by any of the authors."

"This article does not contain any studies with animals performed by any of the authors."

"This article does not contain any studies with human participants or animals performed by any of the 
authors."

\section{Acknowledgements:}

(check) This section is where one or more of the following will be mentioned:

An individual who provided purely technical help.

An individual who helped with editing/proofreading/ writing

A department chair who provided only general support.

Groups of persons who have contributed materially to the paper but whose contributions do not justify authorship may be listed under a heading such as "clinical investigators" or "participating investigators," and their function or contribution should be described - for example, "served as scientific advisors," "critically reviewed the study proposal," "collected data," or "provided and cared for study patients."

For additional information visit the following web site: http://www.icmje.org

Adapted from the editorial office web-site: www.di-ep.com 


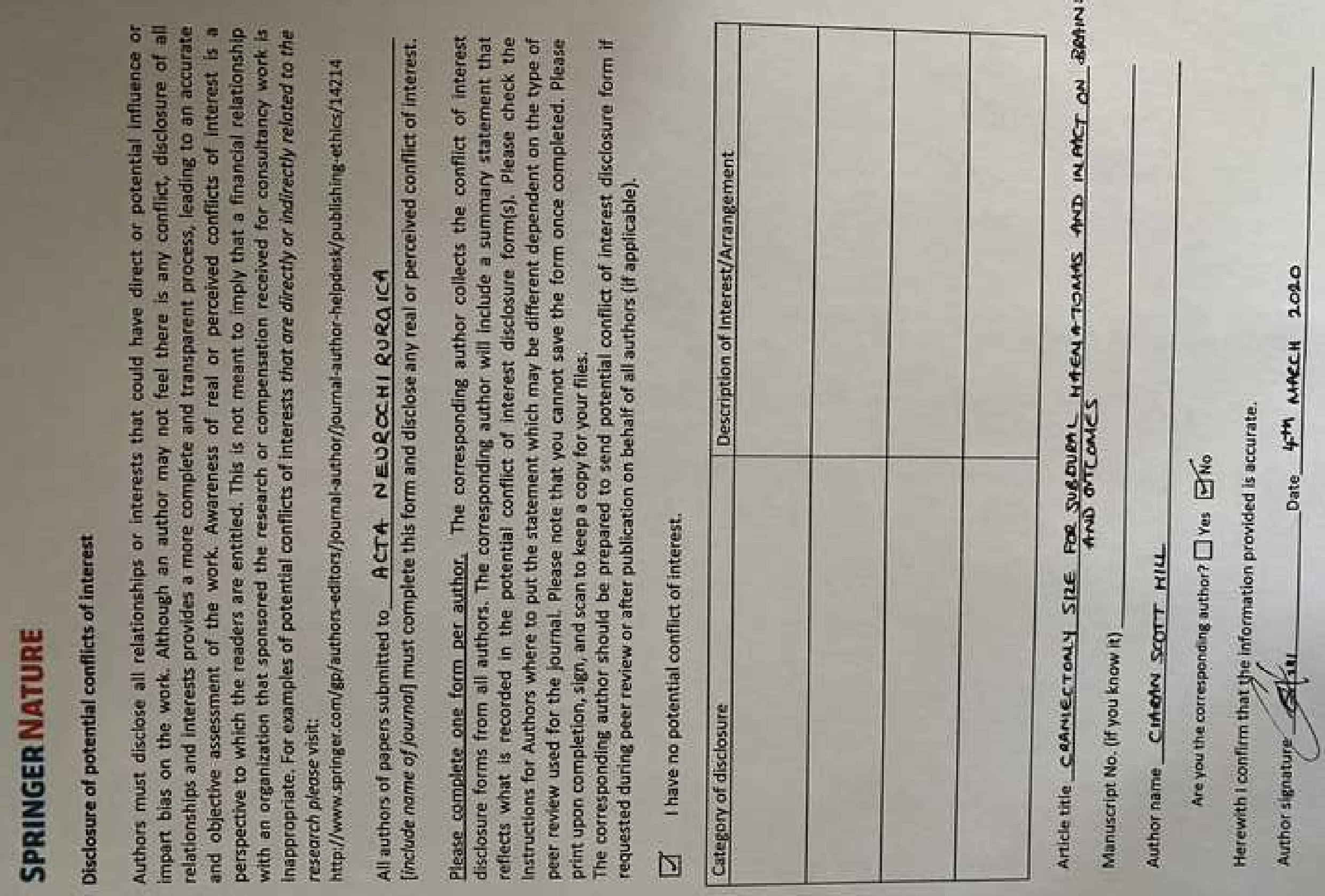




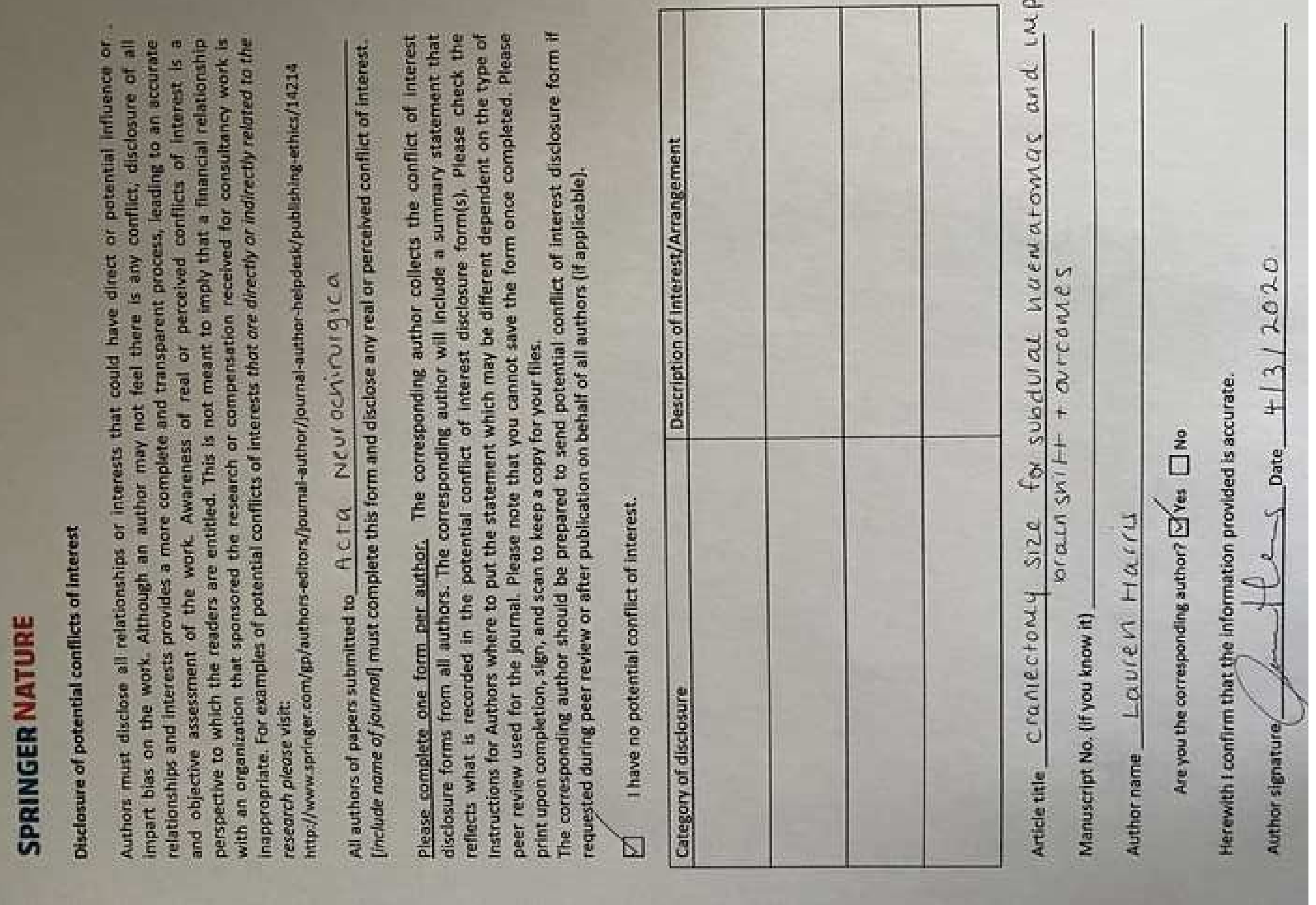




\section{Author response to reviewers' comments}

Thank you for taking the time to review our manuscript. We appreciate your advice and have made the appropriate changes below. We hope it is now of the standard expected for publication in Acta Neurochirurgica. We have answered each of your questions in bold below and have highlighted the changes in red in the text.

Reviewers' comments:

Reviewer \#1: I think the authors need to comment on:

Were all the bone flaps of adequate size - the implication of the paper is that size of bone flap is not important but if all of these are large enough then that may explain the lack of correlation

We have added in Figure 4 to graphically show the size of the craniectomy, and added in an explanation into the discussion (below).

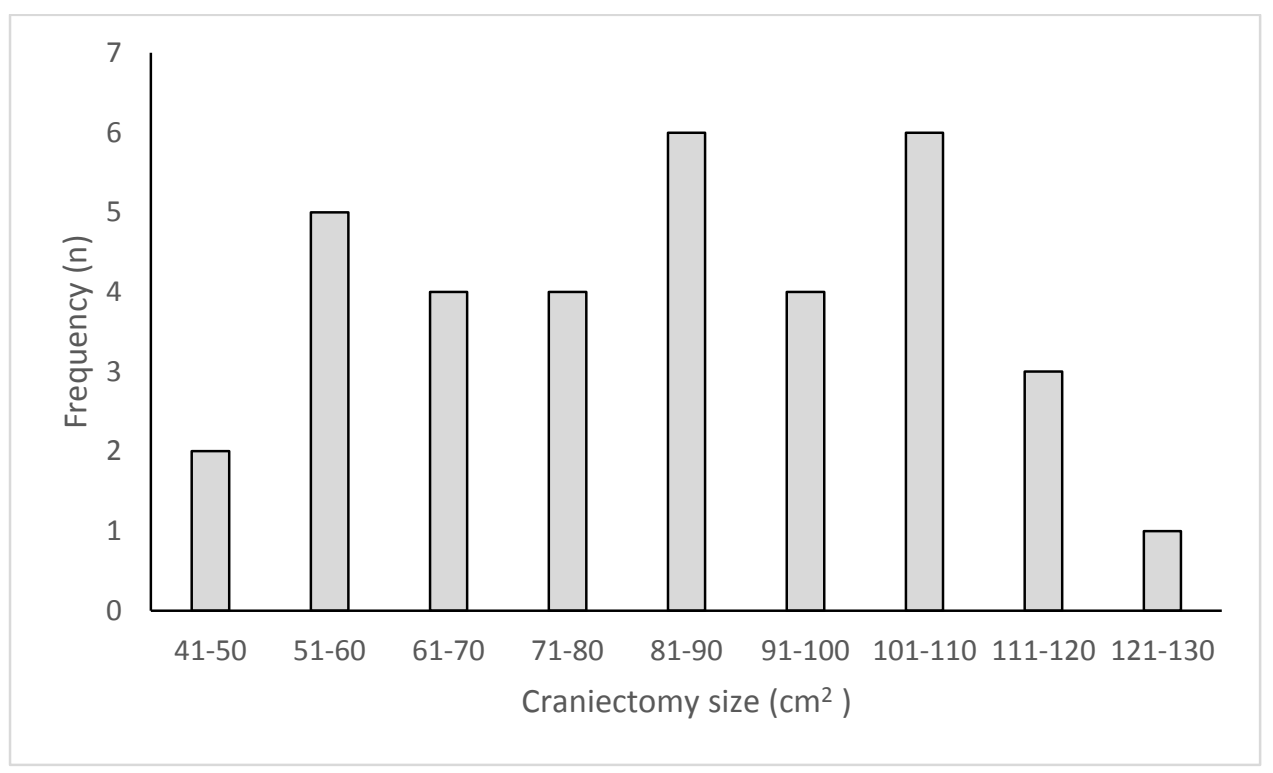

The European Brain Injury Consortium had a mean craniectomy size of $67 \mathrm{~cm}^{2}$ and determined that less than $30 \mathrm{~cm}^{2}$ was inadequate.[8] By this definition, all the bone flaps in our study were of adequate size (Figure 4), which may explain the lack of correlation.

Additionally, not all the cases were planned as primary decompressive craniectomies, some were planned as craniotomies, but swelling and intraoperative appearance may have led to the decision to leave the bone out

Figure 4

Histogram of craniectomy size

$50 \%$ of the patients were mRS of 0 - the implication being that they did not have a particularly severe injury?

On presentation we had over three quarters presenting as a severe TBI, with over half at least one fixed pupil. This is detailed in the results section. We have added in further detail to our discussion to re-iterate this point. We were fortunate to have good outcomes. However, there 
may be some selection bias due to our inclusion criteria - patients required a post-operative CT scan - potentially excluding some bad outcomes. This is highlighted in the text.

The outcomes over a median of $\mathbf{2 3 . 5}$ months were very positive. Despite significant TBI, with patients having a median presentation GCS of 5 (three quarters of the cohort presented as a severe TBI and half had at least one fixed pupil), there were no mortalities and the majority of the patients made a good recovery. Our inclusion criteria necessitated a post-operative CT scan, so patients who were too unstable for a post-operative scan, and may have gone on to die, were excluded.

Reviewer \#2: This is a retrospective study of 36 patients with acute subdural haematomas to see if there is a relationship between craniectomy size and midline shift, brainstem shift and cisternal effacement. It also addresses if sub temporal decompression relates to: midline shift, brainstem shift and cisternal effacement. It also included data on patient outcomes. The results show there is no relationship.

Introduction

1. The reference from the first sentence of the introduction seems to be from an editorial. Would be better to cite primary research for this.

The additional references have been included (ref $17 / 18 / 25$ ).

Jennett B, MacMillan R (1981) Epidemiology of head injury. Br Med J (Clin Res Ed) 282(6258):101-104

Kay A, Teasdale G (2001) Head injury in the United Kingdom. World J Surg 25(9):1210-1220

Teasdale GM (1995) Head injury. J Neurol Neurosurg Psychiatry 58(5):526-539

Materials and Methods

1. States 5 year period. Please give time frame or dates of where this 5 year period resides

\section{Apologies - was stated in the abstract only in error, now added into methods.}

Between June 2008 and August 2013.

2. One would assume patient's with an acute subdural as their primary pathology but also had other brain injuries (e.g. small contusions) are included in the inclusion criteria? Or would these cohort of patients be under the domain of 'diffuse brain injury' and thus excluded? Please clarify here in methods. The results later on infer these pathologies were also included

Patients who underwent craniectomy for an acute subdural haematoma were included irrespective of presence or absence of additional brain injury including contusions or diffuse injury. Patients who underwent bifrontal or bilateral craniectomies were excluded. Clarified as below.

Inclusion criteria were adult patients who underwent a unilateral decompressive craniectomy with a primary diagnosis of acute subdural causing mass effect, including those with evidence of additional brain injury on computed tomography (CT) scan (including contusions $\left(1 \mathrm{~cm}^{3}\right.$ or less), traumatic $\mathrm{SAH}$, extradural haematomas $<1 \mathrm{~cm}$ in thickness). Patients who underwent bifrontal or bilateral craniectomies were excluded. Patients were excluded if they underwent a craniectomy for other causes; including extradural haemorrhage, diffuse brain injury, malignant middle cerebral artery (MCA) infarction, aneurysmal subarachnoid haemorrhage (SAH), or tumour. Other exclusion criteria included patients under the age of 16 , and patients in whom there was no 
pre-operative imaging within six hours of surgery, or post-operative imaging within seven days. This requirement allowed us to calculate the degree of shift or effacement resolution.

3. Please state how to data was stored

All data was stored in accordance with General Data Protection Regulations and the Data Protection Act 2018. It was kept in encrypted pseudo-anonymised files on a computer in a locked room at the hospital. Access was limited to the clinical research team.

Results

1. 36 primary decompressive craniectomies in a 5 year period in London Trauma Centre seems rather small. It would be useful to give a background of the catchment area that neurosurgery serves and the total number of acute subdurals that were operated on.

This is a small number reflecting our strict inclusion and exclusion criteria including a preoperative scan within 6 hours, and a post-operative scan within 7 days. This was necessary to allow us to calculate the degree of shift or effacement resolution as related to the acute subdural and mass effect. We have highlighted this in the results and discussion as below.

The study was carried out in the largest of the four MTCs in London, serving a population of over 2.5 million people.

36 patients underwent a primary decompressive craniectomy for an acute subdural haematoma in a 5-year period between June 2008 and August 2013 and fulfilled our strict inclusion and exclusion criteria.

There are several limitations to this study. It was a retrospective, single centre study, with a relatively small sample size, reflecting out strict inclusion and exclusion criteria.

2. It states time taken between admission and operation was 0 days. Would be more useful to give in number of hours if the mean was 0 days.

\section{Many thanks. Corrected.}

The time taken between admission and operation was a median of 4.2 hours (interquartile range 1.8 hours -1 day).

3. Is there any ICP measurement data available? If so, would be useful to include to see if pre and post op ICP data and compare that with craniectomy size.

Unfortunately, we do not have adequate ICP data available for meaningful inclusion. Records were paper during the study period and we have been unable to retrospectively collect this for the included patients. We have added this into a limitation in the manuscript.

ICP data was unavailable, so we were unable to compare pre and post-operative data and compare it to craniectomy size, or stratify it with pathology. However, brain herniation through the defect acted as a crude surrogate. We hope to assess this in future work.

4. How many patients had a cranioplasty during the follow up? 
Ethical approval

1. Please state if this project was registered as a service evaluation for data collection to commence. Please also state if patient consent was required/obtained to include their radiological imaging.

This study was registered locally with the Clinical Effectiveness Unit as a Service Evaluation prior to data collection. Data was fully anonymised so formal consent was not required.

Tables

1. Table 1. Total number of patients adds up to 35 not 36.1 patient is missing (results state 36 patients)

Apologies - one fall from standing patient was missed from the patients (not the percentage) when erroneously transcribing results from excel. Thank you for highlighting this omission and giving us the opportunity to correct it.

\begin{tabular}{|lll|}
\hline Mechanism & $\begin{array}{l}\text { Patients } \\
(\mathbf{n})\end{array}$ & $\begin{array}{l}\text { Percentage } \\
(\boldsymbol{\%})\end{array}$ \\
\hline Fall from height & 10 & 25.0 \\
Assault & 9 & 27.8 \\
Pedestrian versus vehicle & 6 & 11.1 \\
Other road traffic accident & 4 & 16.7 \\
Fall from standing & 5 & 13.9 \\
Pre-eclampsia & 1 & 2.8 \\
Unknown mechanism & 1 & 2.8 \\
\hline
\end{tabular}

2. Table 4. Total number is again 35 patients in this table not 36.1 patient is missing from the table.

One patient was lost to follow up. This has been made clear in the text and in the description of the table.

Long term follow-up was available in $97.2 \%$ of cases $(35 / 36)$

Table 4: Patient outcomes on the Modified Rankin Scale. Data is given as raw numbers and percent. One patient was lost to follow up.

Editors comment: Please ad data on criteria (inclusion/exclusion) to make a DC and please provide data/discussion to allow stratification for icp/degree of brain swelling and pathology.

Thank you, we have added in the below to reflect your comments.

Methods:

The decision to undergo a decompressive craniectomy was made by the on-call consultant Neurosurgeon in charge of the patient's care, based on the patient's demographics, clinical status, imaging findings and neurotrauma experience. 
Results:

In 17 patients (47.2\%) there was an element of brain herniation through the craniectomy defect on postoperative imaging, as reported by a consultant Neuroradiologist. There was no relationship between brain herniation through the craniectomy defect and pre-operative midline shift $(p=0.679)$, brainstem shift $(p=$ 0.642 ), or craniectomy size ( $p=0.595$ ) on a two-tailed t-test.

33 patients (91.7\%) had a post prognostication Magnetic Resonance Imaging (MRI) scan post-operatively. 20 (60.6\%) had no evidence of DAl on imaging, and 6 (18.2\%), 5 (15.2\%), and 2 (6.1\%) had evidence of grade 1, 2, and 3 DAI respectively.

Discussion:

In $47 \%$ of cases there was herniation of brain through the craniectomy defect on post-operative imaging, suggesting a degree of brain swelling post-operatively. This showed no association with pre-operative shift or craniectomy size.

ICP data was unavailable, so we were unable to compare pre and post-operative data and compare it to craniectomy size, or stratify it with pathology. However, brain herniation through the defect acted as a crude surrogate. We hope to assess this in future work. 


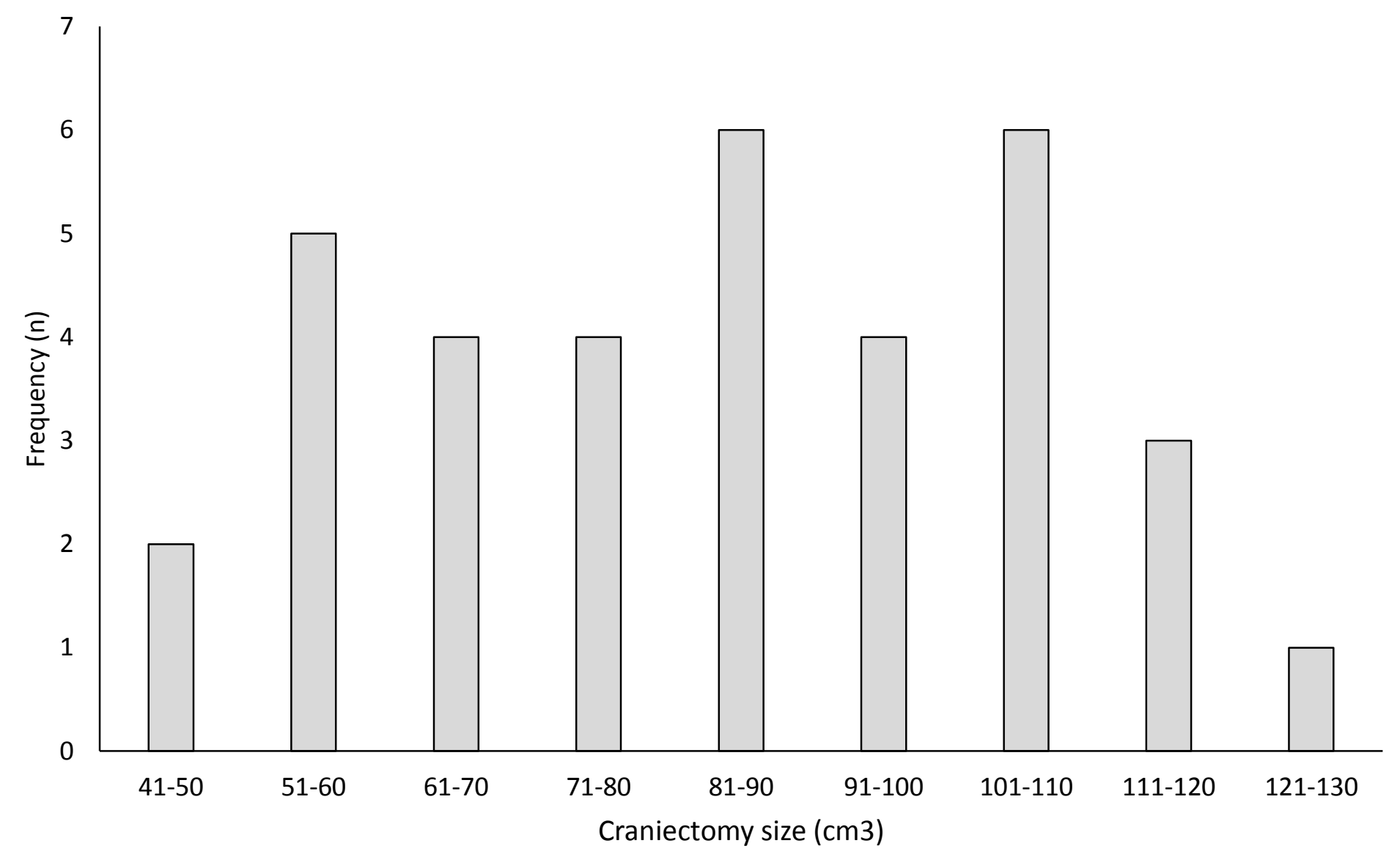




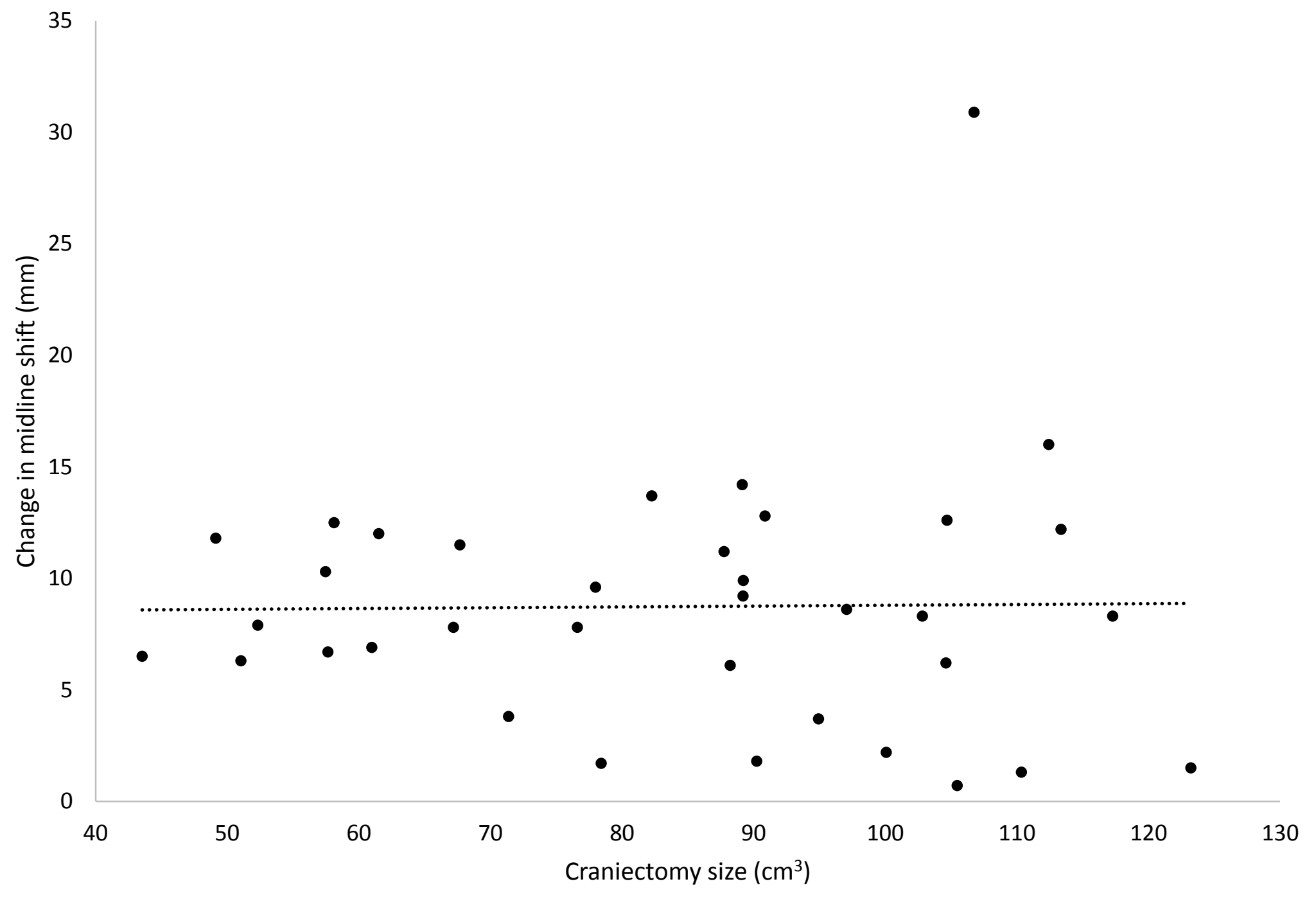




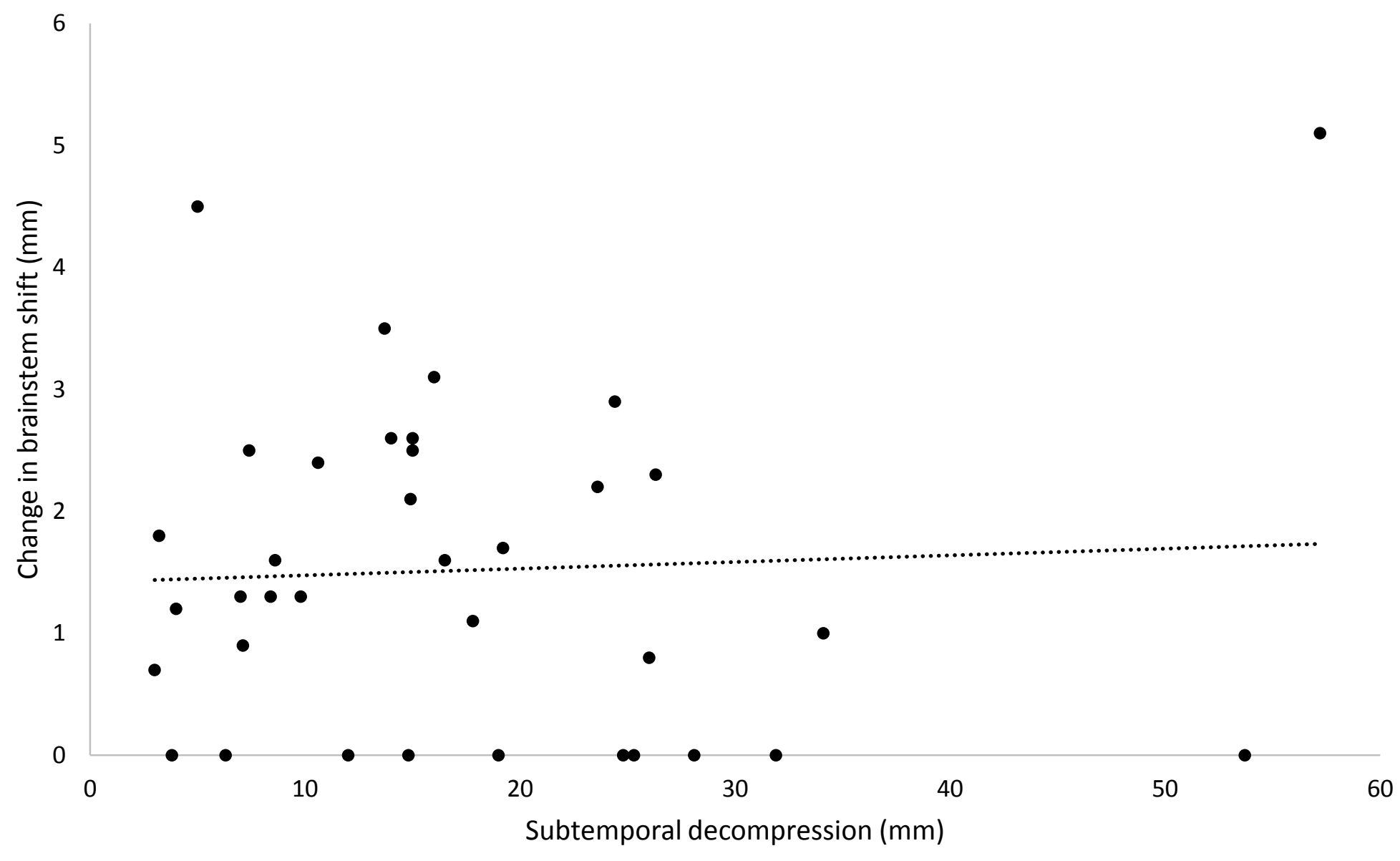




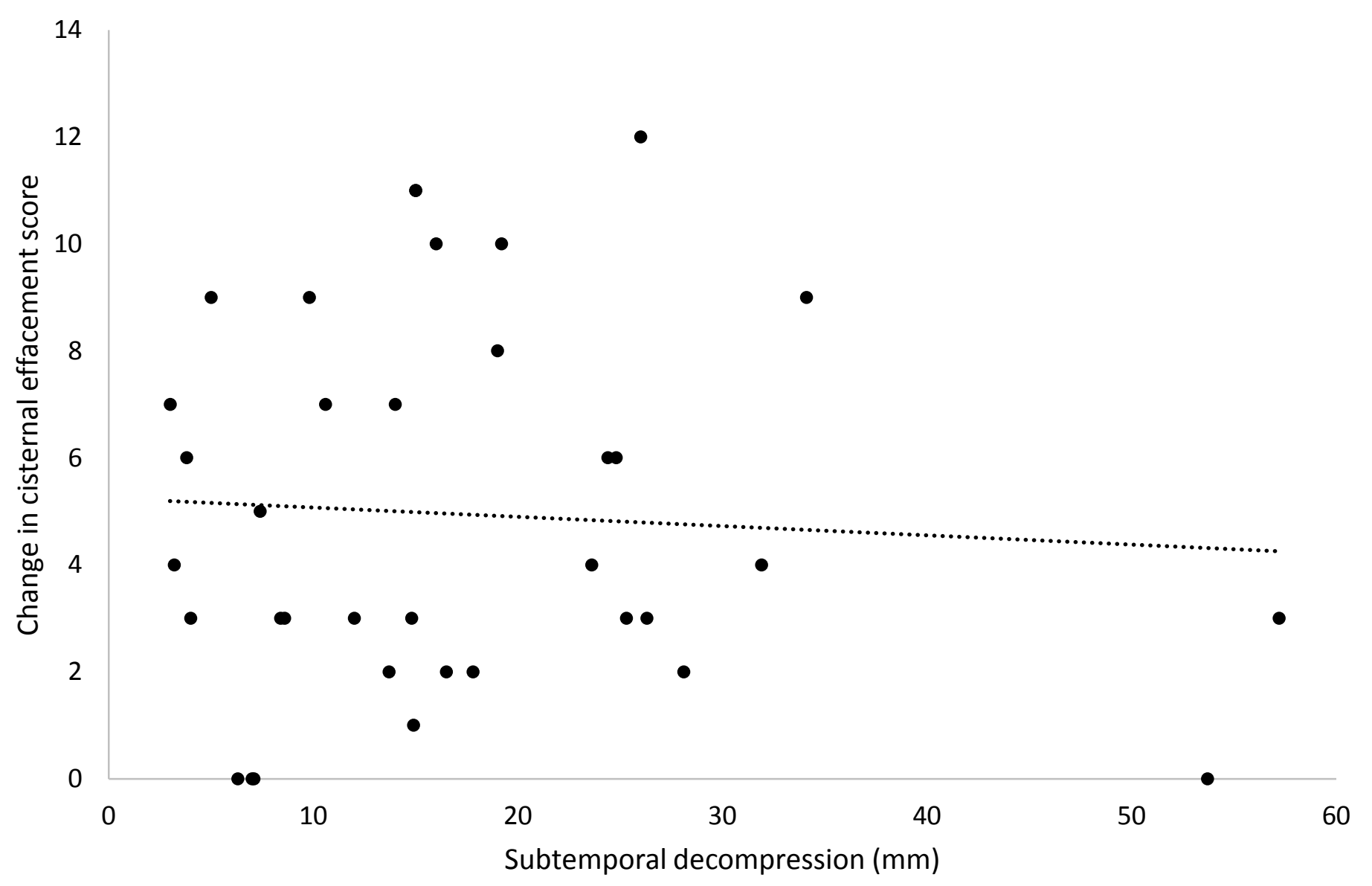



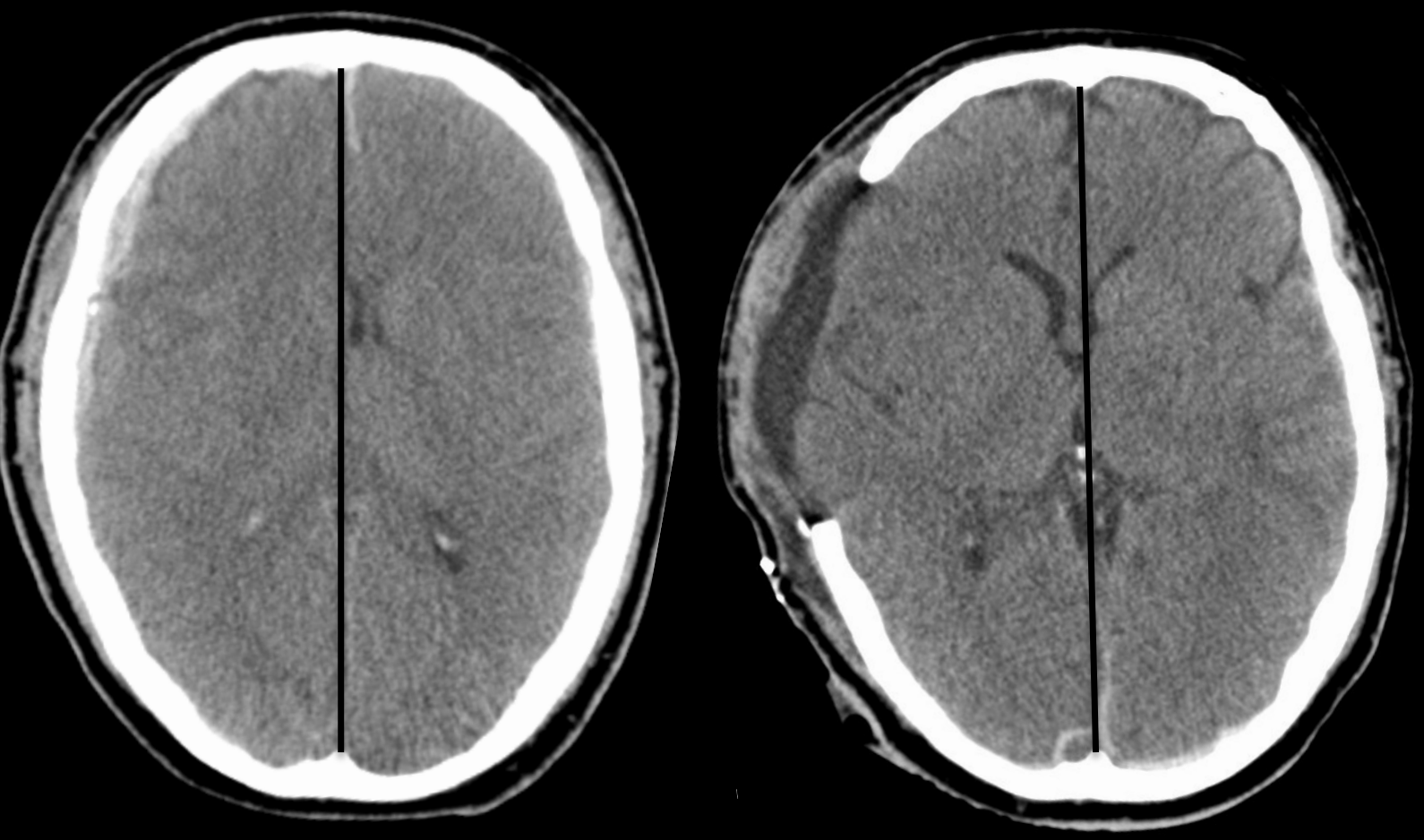


\title{
Title page
}

Craniectomy size for subdural haematomas and the impact on brain shift and outcomes

\author{
Authors: \\ Lauren Harris \\ Department of Neurosurgery, The Royal London Hospital, London, E1 1BB \\ lauren.harris7@nhs.net \\ 02073777000 \\ Ciaran Scott Hill \\ Department of Neurosurgery, The National Hospital for Neurology and Neurosurgery, London, W1B \\ 3GG \\ ciaran.hill@nhs.net
}

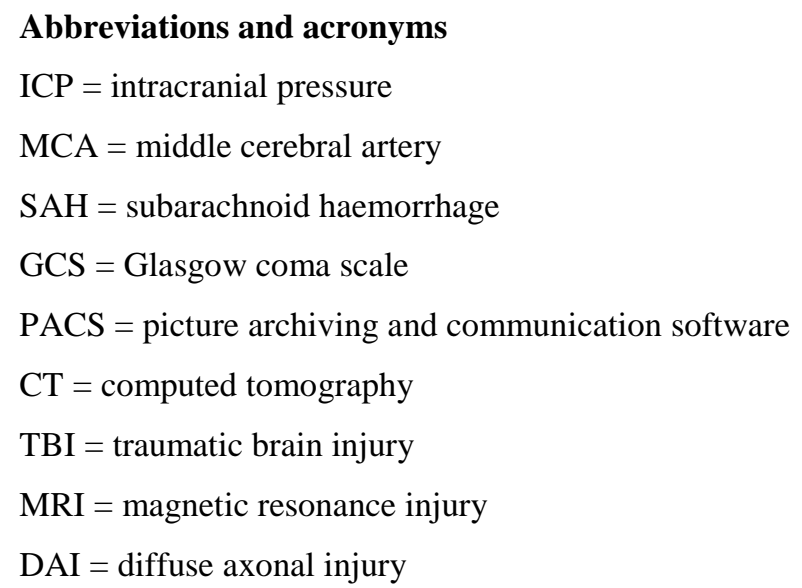




\section{Abstract}

\section{Background}

Midline shift in trauma relates to the severity of head injury. Large craniectomies are thought to help resolve brain shift but can be associated with higher rates of morbidity. This study explores the relationship between craniectomy size and subtemporal decompression for acute subdural haematomas with the resolution of brain compression and outcomes. No systematic study correlating these measures has been reported.

\section{Method}

A retrospective study of all adult cases of acute subdural haematomas that presented to a Major Trauma Centre and underwent a primary decompressive craniectomy between June 2008 and August 2013. Data collection included patient demographics and presentation, imaging findings and outcomes. All imaging metrics were measured by two independent trained assessors. Compression was measured as midline shift, brainstem shift and cisternal effacement.

\section{Results}

36 patients with mean age was $36.1+/-12.5$ (range 16-62) were included, with a median follow-up of 23.5 months (range 2.2-109.6). The median craniectomy size was $88.7 \mathrm{~cm}^{2}$ and the median subtemporal decompression was $15.0 \mathrm{~mm}$. There was significant post-operative resolution of shift as measured by midline shift, brainstem shift and cisternal effacement score (all p<.00001). There was no mortality, and the majority of patients made a good recovery with $82.8 \%$ having a Modified Rankin Score of 2 or less. There was no association between craniectomy size or subtemporal decompression and any markers of brain shift or outcome (all $\left.\mathrm{R}^{2}<0.05\right)$.

\section{Conclusions}

This study suggests that there is no clear relationship between craniectomy size or extent of subtemporal decompression and resolution of brain shift or outcome. Further studies are needed to assess the relative efficacy of large craniectomies and the role of subtemporal decompression.

Keywords: Craniectomy, Decompression, Subdural Hematoma, Shift, Herniation, Brainstem Compression 


\section{Introduction}

Head injury is the leading cause of morbidity and mortality in people aged between one and forty years in the United Kingdom, severely disabling 150-200 people per million annually.[12, 17, 18, 25] Elevated intracranial pressure (ICP) within the fixed-volume skull is one of the most important causes of death and disability, and the management remains a challenge.[4, 26] Primary decompressive craniectomy, when a craniectomy is performed at the time of mass lesion evacuation, may be planned pre- or intraoperatively depending on the degree of brain swelling and ICP. Surgery attempts to reduce global ICP, focal compression and subsequent herniation with the aim to increase survival and reduce morbidity.

Compression is assessed by radiological midline shift at the level of the lateral ventricles and by brainstem compression inferred as the presence or absence of cisternal effacement. The degree of midline shift relates to the severity of the head injury and has been correlated with outcome.[1, 7, 11, 27, 28] Shift in excess of haematoma depth is an additional poor prognostic factor.[21, 23] Although it has not been formally demonstrated it is intuitive that resolution of compression would occur with decompression, and correlate with improved outcome.

This study investigates the results of primary decompressive craniectomy for acute traumatic subdural haematomas. It explores the relationship between craniectomy size and degree of subtemporal decompression with the resolution of compression and outcomes. No systematic study correlating these measures has been reported. The primary aims were (i) does the size of the craniectomy relate to the degree of midline shift, brainstem shift, or cisternal effacement resolution? (ii) does the extent of subtemporal decompression relate to the degree of midline shift, brainstem shift, or cisternal effacement resolution? The secondary aim was to look at the long-term patient outcomes.

\section{Materials and Methods}

A retrospective study of cases of acute subdural haematomas that presented to a Major Trauma Centre (MTC) and underwent a primary decompressive craniectomy in a 5-year period, between June 2008 and August 2013. The study was carried out in the largest of the four MTCs in London, serving a population of over 2.5 million people. Inclusion criteria were adult patients who underwent a unilateral decompressive craniectomy with a primary diagnosis of acute subdural causing mass effect, including those with evidence of additional brain injury on computed tomography (CT) scan (including contusions $\left(1 \mathrm{~cm}^{3}\right.$ or less), traumatic SAH, extradural haematomas $<1 \mathrm{~cm}$ in thickness). Patients who underwent bifrontal or bilateral craniectomies were excluded. Patients were excluded if they underwent a craniectomy for other causes; including extradural haemorrhage, diffuse brain injury, malignant middle cerebral artery (MCA) infarction, aneurysmal subarachnoid haemorrhage (SAH), or tumour. Other exclusion criteria included patients under the age of 16 , and patients in whom there was no pre-operative imaging within six hours of surgery, or post-operative imaging within seven days. This requirement allowed us to calculate the degree of shift or effacement resolution. The decision to undergo a 
decompressive craniectomy was made by the on-call consultant Neurosurgeon in charge of the patient's care, based on the patient's demographics, clinical status, imaging findings and neurotrauma experience.

Data collection included patient demographics, the nature of trauma, admission Glasgow Coma Score (GCS) and pupils, imaging findings (including location of bleed, size of haematoma, contusions, traumatic SAH, brain shift, brainstem compression, craniectomy size, herniation through the craniectomy defect, diffuse axonal injury (DAI)), and outcomes (Modified Rankin Scale, cranioplasty). All imaging metrics were measured using in-house Citrix picture archiving and communication software (PACS). Measurements were undertaken independently by two trained assessors and the mean was then determined. Pre and post-operative CT scans were used. Where there was more than one post-operative scan only the earliest scan was included.

Midline shift was calculated as the displacement of the septum pellucidum in millimetres in an orthogonal plane from the midline. Brainstem shift was calculated as the displacement of the centre point of the midbrain in millimetres in an orthogonal plane from the midline. The midline was defined as a line drawn from the anterior attachment of the falx cerebri on the frontal bone to its posterior attachment on the occipital bone. The centre point of the midbrain was defined as the midpoint of a line drawn from between the cerebral peduncles to between the superior colliculi. Both determination of the midline and measurement of shift were made on the same axial CT slice, at the rostro-caudal midpoint of the lateral ventricle and at the level of the superior colliculi respectively. Haematoma size was calculated as the maximum diameter on axial imaging. Results were averaged and if the deviation between the two assessors' scores were $3 \mathrm{~mm}$ or more for midline shift or $1 \mathrm{~mm}$ or more for brainstem shift then a third assessor took a final measurement. The two measurements that were closest were then retained as an average.

Brainstem compression was assessed using a cisternal effacement score.[3] Measurements were taken at 2 cisternal levels, perimesencephalic at the level of dorsum sellae and $10 \mathrm{~mm}$ above at the level of the quadrigeminal. The perimesencephalic cistern was divided into 4 sections (rhomboid) and the quadrigeminal into 3 (semilunar) (Figure 1 and 2). Each of these 7 segments was scored: $0=$ no effacement, 1 = some effacement, 2 = complete effacement. The total score could range from 0 (no effacement) to 14 (complete effacement). Scores were given by two separate assessors and results averaged.

Craniectomy size was measured using a 3-dimensional reconstruction of the cranium and the volume measurement tool. If the difference in volume measured by the two assessors was greater than $3 \mathrm{~cm}^{2}$, a third assessor recorded an additional measurement and the two closest were retained and averaged.

Extent of subtemporal decompression was measured as the height of the remaining bone from the middle fossa floor to the inferior limit of the craniectomy, typically the lip of squamous temporal bone (Figure 3). It was taken at a point that correlated with the centre of the midbrain using a localizer. This equated 
to a point approximately $2 \mathrm{~cm}$ anterior to the root of the zygoma. The lower the figure the greater the extent of subtemporal decompression, for example a result of zero would be completely flush with the middle fossa floor.

All data was stored in accordance with General Data Protection Regulations and the Data Protection Act 2018. It was kept in encrypted pseudo-anonymised files on a computer in a locked room at the hospital. Access was limited to the clinical research team. Data was analysed with simple statistics, two tailed student t-test, and simple linear regression with Bonferroni correction as appropriate.

\section{Results}

36 patients underwent a primary decompressive craniectomy for an acute subdural haematoma in a 5year period between June 2008 and August 2013 and fulfilled our strict inclusion and exclusion criteria.

The mean age was $36.1+/-12.5$ (range 16-62) and 69.4\% where male (25/36). The mechanism of injury was due to falls, road traffic accidents or assaults in $94.4 \%$ of cases (Table 1). The median GCS at presentation was 5 , with $76.2 \%$ presenting as severe traumatic brain injury (TBI) with a GCS of 3-8, $14.3 \%$ with moderate (GCS 9-13), and 9.5\% with mild TBI (GCS 14-15). Pre-operative pupil response was normal in $46.2 \%$ of cases, unilaterally fixed in $38.4 \%$ and bilaterally fixed and dilated in $15.4 \%$. The time taken between admission and operation was a median of 4.2 hours (interquartile range 1.8 hours 1 day).

There were an equal number of left and right sided convexity haematomas (18 of each). Two patients had an associated smaller extradural, and one patient had bilateral subdural haematomas (the larger left sided subdural was causing the mass effect requiring unilateral craniectomy and hence inclusion in this study). The median size of the haematoma was $8.3 \mathrm{~mm}$, with a midline shift of $8.4 \mathrm{~mm}$ and brainstem shift of $1.65 \mathrm{~mm}$ (Table 2). $63.9 \%$ of patients had contusions on imaging, and $88.9 \%$ had evidence of traumatic SAH blood.

The median craniectomy size was $88.7 \mathrm{~cm}^{2}$ (range $43.5-123.2 \mathrm{~cm}^{2}$ ) (Figure 4) and the median subtemporal decompression was $15.0 \mathrm{~mm}$ (range $3.0-57.2 \mathrm{~mm}$ ). There was no association with either the size of craniectomy or subtemporal decompression with the change in midline shift, brainstem shift or cisternal effacement score (Table 3). This can be seen graphically in Figures 5-7. In 17 patients (47.2\%) there was an element of brain herniation through the craniectomy defect on post-operative imaging, as reported by a consultant Neuroradiologist. There was no relationship between brain herniation through the craniectomy defect and pre-operative midline shift $(\mathrm{p}=0.679)$, brainstem shift $(\mathrm{p}=0.642)$, or craniectomy size $(\mathrm{p}=0.595)$ on a two-tailed t-test.

33 patients $(91.7 \%)$ had a post prognostication Magnetic Resonance Imaging (MRI) scan postoperatively. $20(60.6 \%)$ had no evidence of DAI on imaging, and $6(18.2 \%), 5(15.2 \%)$, and $2(6.1 \%)$ had evidence of grade 1,2 , and 3 DAI respectively. 
Long term follow-up was available in $97.2 \%$ of cases (35/36) with a median of 23.5 months (range 2.2 -109.6 months). There was no mortality in this cohort. The majority of the patients made a good recovery with $82.8 \%$ having a favourable modified Rankin score of 2 or less (Table 4). Additionally, there was no association between craniectomy size or subtemporal decompression and outcome $\left(\mathrm{R}^{2}=0.0092\right.$ and 0.0487 respectively). $100 \%$ of patients had a cranioplasty at a median of 9.3 months (range $1.6-53.1$ months).

\section{Discussion}

This study demonstrates a statistically significant reduction in post-operative midline shift, brainstem shift and cisternal effacement following primary craniectomy for acute subdural haematoma evacuation. However, the size of craniectomy or extent of subtemporal decompression showed a striking lack of correlation with the degree of midline shift, brainstem shift or cisternal effacement resolution. In this study, the size of the craniectomy and subtemporal decompression was patient and surgeon specific, with the aim to resolve midline shift and brainstem compression, and improve patient outcomes. This gave us the opportunity to pragmatically correlate different sizes of craniectomy with subtypes of brain shift.

Despite being one of the oldest and most common cranial neurosurgical procedures the exact indications and rationale for a primary craniectomy are still being elucidated. A large craniectomy with a subtemporal decompression is not a benign procedure and should only be undertaken if there is likely to be a benefit to the patient. Evidence suggests that primary decompressive craniectomy is performed in a significant proportion of acute subdural haematoma evacuations in the UK.[20] The decision to perform a craniectomy must be balanced against several negative consequences. There is the risk of surgical complication and morbidity, a cosmetic bony defect as well as risk of damage to the temporalis muscle/innervation, and the potential need for a later operation to insert a cranioplasty which itself carries additional risks including infection.[6, 14, 15, 24] It is important to know if the craniectomy size correlates with shift resolution, to determine if there is a critical size required, and to help decide if a craniotomy will suffice. The benefit of a primary craniectomy versus craniotomy for acute subdural haematomas is uncertain, and is being investigated in the RESCUE-ASDH trial.[19, 22]

The finding that craniectomy size does not correlate with the degree of midline shift can reasonably be explained by the assertion that the primary mediators of midline shift are a) residual haematoma and b) brain swelling and underlying parenchymal injury. The European Brain Injury Consortium had a mean craniectomy size of $67 \mathrm{~cm}^{2}$ and determined that less than $30 \mathrm{~cm}^{2}$ was inadequate.[8] By this definition, all the bone flaps in our study were of adequate size (Figure 4), which may explain the lack of correlation. Decompression is a secondary factor and any role it plays in resolution of midline shift did not show a correlation. It may be the case that more swollen brains, and worse injuries, received larger flaps, essentially masking any effect on reducing shift. Additionally, not all the cases were planned as primary decompressive craniectomies, some were planned as craniotomies, but swelling and intraoperative 
appearance may have led to the decision to leave the bone out. The confounding role of a progression in pathological process in the intervening time between the preoperative and postoperative scans also cannot be discounted. In $47 \%$ of cases there was herniation of brain through the craniectomy defect on post-operative imaging, suggesting a degree of brain swelling post-operatively. This showed no association with pre-operative shift or craniectomy size.

Subtemporal decompression was described by Harvey Cushing as a method of controlling pressure resulting from brain tumours and traumatic blast injuries.[9, 10] It was initially used as an isolated method without the larger craniectomy seen with modern decompressions.[2, 13, 16] Today, a craniectomy including the subtemporal decompression is common practice. However, it has associated risks, including bleeding (particularly with skull base fractures), need for a preauricular incision, prolonged operative time, lateral brain herniation, poor cosmesis and the risk of damaging the frontalis branch of the facial nerve. It is important to determine whether subtemporal decompression improves brain shift or cisternal effacement before continuing to routinely perform this additional step.

The theory behind subtemporal decompression is that any pressure generated at the middle fossa floor, for example due to brain swelling or contusions, will be transferred medially to the brainstem in the form of uncal herniation if there is not a sufficiently low craniectomy to allow expansion of the mass laterally. Although an intuitively appealing theory this has never been empirically tested. The subtemporal region is not a true 'closed box' with only a medial opening. Although there are bone limits anteriorly (greater wing of sphenoid), laterally (squamous temporal bone), inferiorly (fossa floor) and posteriorly (petrous ridge), the fossa is largely open superiorly apart from an anterior portion of the temporal pole covered by the lesser wing of sphenoid. This means that pressure transfer can occur from the middle fossa floor superiorly to the cerebral hemisphere, and vice versa. Whether pressure shifts brain medially or superiorly from the middle fossa will depend on the pressure differential within these spaces. By 'opening the box' with a craniectomy above the middle temporal fossa it may be the case that this route of pressure equalization is adequate to lead to a resolution in brainstem shift and cisternal effacement. The contribution of the subtemporal portion of this decompression was not evident in this study and it may be that it contributes far less to resolution of pressure on the brainstem than classically taught. Another factor to consider is the pliability of the skin and soft tissue lateral to the middle fossa after closure, in comparison to the convexity. The extra bulk of temporalis and tension provided by proximity to bone attachments may make expansion here less likely to occur, particularly in the context of a large hemispheric decompression.

The outcomes over a median of 23.5 months were very positive. Despite significant TBI, with patients having a median presentation GCS of 5 (three quarters of the cohort presented as a severe TBI and half had at least one fixed pupil), there were no mortalities and the majority of the patients made a good recovery. Our inclusion criteria necessitated a post-operative CT scan, so patients who were too unstable for a post-operative scan, and may have gone on to die, were excluded. In addition, as over $85 \%$ of our cohort had good outcomes, we found no association between pre-operative compression or craniectomy 
size and outcome. This is likely to be a result of too few patients having poor outcomes for conclusions to be reliably drawn.

There are several limitations to this study. It was a retrospective, single centre study, with a relatively small sample size, reflecting out strict inclusion and exclusion criteria. We were unable to elucidate whether the craniectomy was planned preoperatively or intraoperatively. A decision may provisionally be made on the preoperative scan but the final decision is usually made after the state of the brain and the space available after clot evacuation has been visualised. We were unable to assess whether the bone opening was increased intraoperatively, for example, as a result of unexpected brain swelling, and postoperative pupil response documentation was poor. ICP data was unavailable, so we were unable to compare pre and post-operative data and compare it to craniectomy size, or stratify it with pathology. However, brain herniation through the defect acted as a crude surrogate. We hope to assess this in future work.

We would argue that knowledge of these factors was not necessary to determine a correlation between extent of craniectomy and shift. This was a pragmatic analysis and demonstrated the real-life correlation between craniectomy size and various subtypes of brain shift. The lack of a clear linear relationship, or even a correlation, between craniectomy size and markers of brain shift urges us to re-examine the importance of the bone opening in a craniectomy for evacuation of subdural haematomas.

\section{Conclusion}

This study suggests that there is no clear relationship between craniectomy size or extent of subtemporal decompression and resolution of brain shift or outcome. This includes midline shift at the level of the foramen of Monro, shift at the level of the midbrain and cisternal effacement. Other factors, including adequacy of haematoma evacuation, surgeon's prediction of subsequent brain swelling, and the heterogenous nature of TBI, are likely to be critical.[5] Further studies should focus on larger prospective series or randomised controlled studies to assess the relative efficacy of large craniectomies and the role of subtemporal decompression.

\section{Funding}

No funding was received for this research.

\section{Conflict of Interest}

All authors certify that they have no affiliations with or involvement in any organization or entity with any financial interest (such as honoraria; educational grants; participation in speakers' bureaus; membership, employment, consultancies, stock ownership, or other equity interest; and expert testimony or patent-licensing arrangements), or non-financial interest (such as personal or professional 
relationships, affiliations, knowledge or beliefs) in the subject matter or materials discussed in this manuscript.

\section{Ethical approval}

This study was registered locally with the Clinical Effectiveness Unit as a Service Evaluation prior to data collection. Data was fully anonymised so formal consent was not required.

\section{Acknowledgements}

We would like to thank Angelos Triantafyllidis and Vejay Vakharia who helped with data collection.

\section{Figures}

Figure 1

CT scan at level of perimesencephalic cistern demonstrating the 4 borders used to calculate cisternal effacement score.

Figure 2

CT scan at level of quadrigeminal cistern demonstrating the 3 borders used to calculate the cisternal effacement score.

\section{Figure 3}

CT with bone windows demonstrating a subtemporal decompression as measured in the coronal plane.

\section{Figure 4 \\ Histogram of craniectomy size}

\section{Figure 5}

Graph to show the change in midline shift in relation to craniectomy size

\section{Figure 6}

Graph to show the change in brainstem shift in relation to subtemporal decompression

\section{Figure 7}

Graph to show the change in cisternal effacement in relation to subtemporal decompression

Figure 8

CT brain showing pre and post-operative midline shift

\section{Tables}




\begin{tabular}{|lll|}
\hline Mechanism & $\begin{array}{l}\text { Patients } \\
(\mathbf{n})\end{array}$ & $\begin{array}{l}\text { Percentage } \\
(\boldsymbol{\%})\end{array}$ \\
\hline Fall from height & 10 & 25.0 \\
Assault & 9 & 27.8 \\
Pedestrian versus vehicle & 6 & 11.1 \\
Other road traffic accident & 4 & 16.7 \\
Fall from standing & 5 & 13.9 \\
Pre-eclampsia & 1 & 2.8 \\
Unknown mechanism & 1 & 2.8 \\
\hline
\end{tabular}

Table 1: The mechanism of injury for acute subdural haemorrhage.

\begin{tabular}{|llll|}
\hline Variable & Pre-operative & Post-operative & p value \\
& & & \\
\hline Haematoma size $(\mathrm{mm})$ & $8.3(3.1-32.3)$ & & \\
Septum pellucidum midline shift $(\mathrm{mm})$ & $8.4(0-18.8)$ & $0.45(-12.1-12.1)$ & $<.00001$ \\
Brainstem shift (mm) & $1.65(0-5.1)$ & $0(-2.6-3)$ & $<.00001$ \\
Cistern effacement score & $6(0-14)$ & $0.5(0-12)$ & $<.00001$ \\
\hline
\end{tabular}

Table 2: Pre and post-operative imaging measurements, including haematoma size, septum pellucidum midline shift, brainstem shift and cistern effacement score. Results given as median with range in brackets. $P$ values calculated with two tailed t-test. All p values are statistically significant.

\begin{tabular}{|ll|}
\hline Association & $\mathbf{R}^{\mathbf{2}}$ \\
\hline Craniectomy size and: & 0.0002 \\
Midline shift & 0.0133 \\
Brainstem shift & 0.049 \\
Cisternal effacement score & \\
& \\
Subtemporal decompression and: & \\
Midline shift & 0.0218 \\
Brainstem shift & 0.0008 \\
Cisternal effacement score & 0.0001 \\
\hline
\end{tabular}

Table 3: Association of craniectomy size and subtemporal decompression with the change in midline shift, brain stem shift and cisternal effacement score. Correlation co-efficient $\left(R^{2}\right)$ calculated with linear regression.

\begin{tabular}{|l|l|}
\hline Modified Rankin Scale & $\begin{array}{l}\text { Patients } \\
\mathbf{n}(\%)\end{array}$ \\
\hline 6 - death & 0 \\
\hline $5-$ severe disability & 0 \\
\hline
\end{tabular}




\begin{tabular}{|l|l|}
\hline $4-$ moderate severe disability & $5(14.3)$ \\
\hline $3-$ moderate disability & $1(2.9)$ \\
\hline $2-$ slight disability & $4(11.4)$ \\
\hline $1-$ no significant disability & $9(25.7)$ \\
\hline $0-$ no symptoms & $16(45.7)$ \\
\hline
\end{tabular}

Table 4: Patient outcomes on the Modified Rankin Scale. Data is given as raw numbers and percent. One patient was lost to follow up.

\section{References}

1. Akbik OS, Starling R V., Gahramanov S, Zhu Y, Lewis J (2019) Mortality and Functional Outcome in Surgically Evacuated Acute Subdural Hematoma in Elderly Patients. World Neurosurg 126:e1235-e1241

2. Alexander E, Ball MR, Laster DW (1987) Subtemporal decompression: Radiological observations and Current surgical experience. Br J Neurosurg 1(4):427-433

3. Artru F, Jourdan C, Convert J, Deschamps J, Guichard P, Chiara Y, Terrier A, Deleuze R, Duquesnel J (1989) An effacement score for basal cisterns to predict ICP level and outcome after closed head injury. Intracranial Press VII 29(4):273-279

4. Balestreri M, Czosnyka M, Hutchinson P, Steiner LA, Hiler M, Smielewski P, Pickard JD (2006) Impact of intracranial pressure and cerebral perfusion pressure on severe disability and mortality after head injury. Neurocrit Care 4(1):8-13

5. Bor-Seng-Shu E, De-Lima-Oliveira M, Nogueira RC, Almeida KJ, Paschoal EHA, Paschoal FM (2019) Decompressive craniectomy for traumatic brain injury: Postoperative TCD cerebral hemodynamic evaluation. Front Neurol. doi: 10.3389/fneur.2019.00354

6. Cabraja M, Klein M, Lehmann TN (2009) Long-term results following titanium cranioplasty of large skull defects. Neurosurg Focus 26(6):1-7

7. Chiewvit P, Tritakarn S, Nanta-aree S, Suthipongchai S (2010) Degree of midline shift from CT scan predicted outcome in patients with head injuries. J Med Assoc Thai 93(1):99-107

8. Compagnone C, Murray GD, Teasdale GM, Maas AIR, Esposito D, Princi P, D'Avella D, Servadei F (2005) The management of patients with intradural post-traumatic mass lesions: A multicenter survey of current approaches to surgical management in 729 patients coordinated by the European Brain Injury Consortium. Neurosurgery 57(6):1183-1191

9. Cushing H (1905) The establishment of cerebral hernia as a decompressive measure for inaccessible brain tumors : with the description of intermuscular methods of making the bone defect in temporal and occipital. Surgery, Gynecol Obstet 1(October):106-123

10. CUSHING H (1908) SUBTEMPORAL DECOMPRESSIVE OPERATIONS FOR THE INTRACRANIAL COMPLICATIONS ASSOCIATED WITH BURSTING FRACTURES OF THE SKULL. Ann Surg 47(5):641-644

11. Fearnside MR, Cook RJ, Mcdougall P, Mcneil RJ (1993) The Westmead head injury project outcome in severe head injury. A comparative analysis of pre-hospital, clinical and CT 
variables. Br J Neurosurg 7(3):267-279

12. Fleminger S, Ponsford J (2005) Long term outcome after traumatic brain injury. Br Med J 331(7530):1419-1420

13. Gower DJ, Lee KS, McWhorter JM, Pitts LH, Geisler FH (1988) Role of subtemporal decompression in severe closed head injury. Neurosurgery 23(4):417-422

14. Hill CS, Luoma AMV, Wilson SR, Kitchen N (2012) Titanium cranioplasty and the prediction of complications. Br J Neurosurg 26(6):832-837

15. Honeybul S, Ho KM (2014) Decompressive craniectomy for severe traumatic brain injury: The relationship between surgical complications and the prediction of an unfavourable outcome. Injury 45(9):1332-1339

16. Huang X, Wen L (2010) Technical considerations in decompressive craniectomy in the treatment of traumatic brain injury. Int J Med Sci 7(6):385-390

17. Jennett B, MacMillan R (1981) Epidemiology of head injury. Br Med J (Clin Res Ed) 282(6258):101-104

18. Kay A, Teasdale G (2001) Head injury in the United Kingdom. World J Surg 25(9):1210-1220

19. Kolias AG, Li LM, Guilfoyle MR, Timofeev I, Corteen EA, Pickard JD, Kirkpatrick PJ, Menon DK, Hutchinson PJ (2013) Decompressive craniectomy for acute subdural hematomas: Time for a randomized trial. Acta Neurochir (Wien) 155(1):187-188

20. Kolias AG, Scotton WJ, Belli A, et al (2013) Surgical management of acute subdural haematomas: Current practice patterns in the United Kingdom and the Republic of Ireland. Br J Neurosurg 27(3):330-333

21. Moussa WMM, Khedr WM, Elwany AH (2018) Prognostic significance of hematoma thickness to midline shift ratio in patients with acute intracranial subdural hematoma: a retrospective study. Neurosurg Rev 41(2):483-488

22. Phan K, Moore JM, Griessenauer C, Dmytriw AA, Scherman DB, Sheik-Ali S, Adeeb N, Ogilvy CS, Thomas A, Rosenfeld J V. (2017) Craniotomy Versus Decompressive Craniectomy for Acute Subdural Hematoma: Systematic Review and Meta-Analysis. World Neurosurg 101:677-685.e2

23. Quattrocchi KB, Prasad P, Willits NH, Wagner FC (1991) Quantification of midline shift as a predictor of poor outcome following head injury. Surg Neurol 35(3):183-8

24. Reid Gooch M, Gin GE, Kenning TJ, German JW (2009) Complications of cranioplasty following decompressive craniectomy: Analysis of 62 cases. Neurosurg Focus 26(6):1-7

25. Teasdale GM (1995) Head injury. J Neurol Neurosurg Psychiatry 58(5):526-539

26. Vibbert M, Mayer SA (2010) Early decompressive hemicraniectomy following malignant ischemic stroke: The crucial role of timing. Curr Neurol Neurosci Rep 10(1):1-3

27. Yılmaz İ, Ertem DH, Kılıç M, Altaş K, Mirhasilova M, Özdemir B, Tanrıverdi O, Yılmaz A (2019) Factors associated with mortality in acute subdural hematoma: Is decompressive craniectomy effective? Ulus Travma ve Acil Cerrahi Derg 25(2):147-153

28. Zumkeller M, Behrmann R, Heissler HE, Dietz H (1996) Computed tomographic criteria and survival rate for patients with acute subdural hematoma. Neurosurgery 39(4):708-713 
1

2

3

4

7

8

10

11

12

13

14

15

16

17

18

19

20

21

22

23

24

25

26

27

28

29

30

31

32

33

34

35

36

37

38

39

40

41

42

43

44

45

46

47

48

49

50

51

52

53

54

55

56

57

58

59

60

61

62

63

64

65 


\title{
Title page
}

Craniectomy size for subdural haematomas and the impact on brain shift and outcomes

\author{
Authors: \\ Lauren Harris \\ Department of Neurosurgery, The Royal London Hospital, London, E1 1BB \\ lauren.harris7@nhs.net \\ 02073777000 \\ Ciaran Scott Hill \\ Department of Neurosurgery, The National Hospital for Neurology and Neurosurgery, London, W1B \\ $3 \mathrm{GG}$ \\ ciaran.hill@nhs.net
}

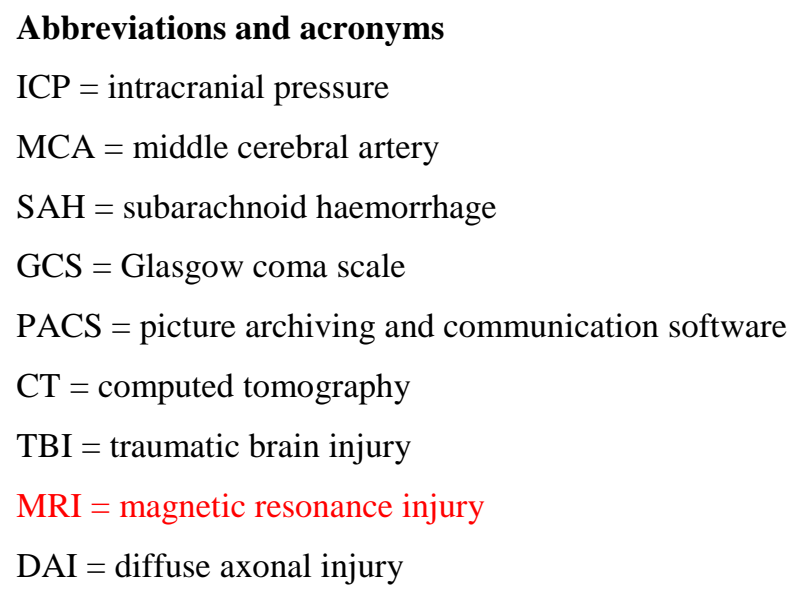




\section{Abstract}

\section{Background}

Midline shift in trauma relates to the severity of head injury. Large craniectomies are thought to help resolve brain shift but can be associated with higher rates of morbidity. This study explores the relationship between craniectomy size and subtemporal decompression for acute subdural haematomas with the resolution of brain compression and outcomes. No systematic study correlating these measures has been reported.

\section{Method}

A retrospective study of all adult cases of acute subdural haematomas that presented to a Major Trauma Centre and underwent a primary decompressive craniectomy between June 2008 and August 2013. Data collection included patient demographics and presentation, imaging findings and outcomes. All imaging metrics were measured by two independent trained assessors. Compression was measured as midline shift, brainstem shift and cisternal effacement.

\section{Results}

36 patients with mean age was $36.1+/$ - 12.5 (range 16-62) were included, with a median follow-up of 23.5 months (range 2.2-109.6). The median craniectomy size was $88.7 \mathrm{~cm}^{2}$ and the median subtemporal decompression was $15.0 \mathrm{~mm}$. There was significant post-operative resolution of shift as measured by midline shift, brainstem shift and cisternal effacement score (all p<.00001). There was no mortality, and the majority of patients made a good recovery with $82.8 \%$ having a Modified Rankin Score of 2 or less. There was no association between craniectomy size or subtemporal decompression and any markers of brain shift or outcome (all $\left.\mathrm{R}^{2}<0.05\right)$.

\section{Conclusions}

This study suggests that there is no clear relationship between craniectomy size or extent of subtemporal decompression and resolution of brain shift or outcome. Further studies are needed to assess the relative efficacy of large craniectomies and the role of subtemporal decompression.

Keywords: Craniectomy, Decompression, Subdural Hematoma, Shift, Herniation, Brainstem Compression 


\section{Introduction}

Head injury is the leading cause of morbidity and mortality in people aged between one and forty years in the United Kingdom, severely disabling 150-200 people per million annually.[12, 17, 18, 25] Elevated intracranial pressure (ICP) within the fixed-volume skull is one of the most important causes of death and disability, and the management remains a challenge.[4, 26] Primary decompressive craniectomy, when a craniectomy is performed at the time of mass lesion evacuation, may be planned pre- or intraoperatively depending on the degree of brain swelling and ICP. Surgery attempts to reduce global ICP, focal compression and subsequent herniation with the aim to increase survival and reduce morbidity.

Compression is assessed by radiological midline shift at the level of the lateral ventricles and by brainstem compression inferred as the presence or absence of cisternal effacement. The degree of midline shift relates to the severity of the head injury and has been correlated with outcome.[1, 7, 11, 27, 28] Shift in excess of haematoma depth is an additional poor prognostic factor.[21, 23] Although it has not been formally demonstrated it is intuitive that resolution of compression would occur with decompression, and correlate with improved outcome.

This study investigates the results of primary decompressive craniectomy for acute traumatic subdural haematomas. It explores the relationship between craniectomy size and degree of subtemporal decompression with the resolution of compression and outcomes. No systematic study correlating these measures has been reported. The primary aims were (i) does the size of the craniectomy relate to the degree of midline shift, brainstem shift, or cisternal effacement resolution? (ii) does the extent of subtemporal decompression relate to the degree of midline shift, brainstem shift, or cisternal effacement resolution? The secondary aim was to look at the long-term patient outcomes.

\section{Materials and Methods}

A retrospective study of cases of acute subdural haematomas that presented to a Major Trauma Centre (MTC) and underwent a primary decompressive craniectomy in a 5-year period, between June 2008 and August 2013. The study was carried out in the largest of the four MTCs in London, serving a population of over 2.5 million people. Inclusion criteria were adult patients who underwent a unilateral decompressive craniectomy with a primary diagnosis of acute subdural causing mass effect, including those with evidence of additional brain injury on computed tomography (CT) scan (including contusions $\left(1 \mathrm{~cm}^{3}\right.$ or less), traumatic SAH, extradural haematomas $<1 \mathrm{~cm}$ in thickness). Patients who underwent bifrontal or bilateral craniectomies were excluded. Patients were excluded if they underwent a craniectomy for other causes; including extradural haemorrhage, diffuse brain injury, malignant middle cerebral artery (MCA) infarction, aneurysmal subarachnoid haemorrhage (SAH), or tumour. Other exclusion criteria included patients under the age of 16 , and patients in whom there was no pre-operative imaging within six hours of surgery, or post-operative imaging within seven days. This requirement allowed us to calculate the degree of shift or effacement resolution. The decision to undergo a 
decompressive craniectomy was made by the on-call consultant Neurosurgeon in charge of the patient's care, based on the patient's demographics, clinical status, imaging findings and neurotrauma experience.

Data collection included patient demographics, the nature of trauma, admission Glasgow Coma Score (GCS) and pupils, imaging findings (including location of bleed, size of haematoma, contusions, traumatic SAH, brain shift, brainstem compression, craniectomy size, herniation through the craniectomy defect, diffuse axonal injury (DAI)), and outcomes (Modified Rankin Scale, cranioplasty). All imaging metrics were measured using in-house Citrix picture archiving and communication software (PACS). Measurements were undertaken independently by two trained assessors and the mean was then determined. Pre and post-operative CT scans were used. Where there was more than one post-operative scan only the earliest scan was included.

Midline shift was calculated as the displacement of the septum pellucidum in millimetres in an orthogonal plane from the midline. Brainstem shift was calculated as the displacement of the centre point of the midbrain in millimetres in an orthogonal plane from the midline. The midline was defined as a line drawn from the anterior attachment of the falx cerebri on the frontal bone to its posterior attachment on the occipital bone. The centre point of the midbrain was defined as the midpoint of a line drawn from between the cerebral peduncles to between the superior colliculi. Both determination of the midline and measurement of shift were made on the same axial CT slice, at the rostro-caudal midpoint of the lateral ventricle and at the level of the superior colliculi respectively. Haematoma size was calculated as the maximum diameter on axial imaging. Results were averaged and if the deviation between the two assessors' scores were $3 \mathrm{~mm}$ or more for midline shift or $1 \mathrm{~mm}$ or more for brainstem shift then a third assessor took a final measurement. The two measurements that were closest were then retained as an average.

Brainstem compression was assessed using a cisternal effacement score.[3] Measurements were taken at 2 cisternal levels, perimesencephalic at the level of dorsum sellae and $10 \mathrm{~mm}$ above at the level of the quadrigeminal. The perimesencephalic cistern was divided into 4 sections (rhomboid) and the quadrigeminal into 3 (semilunar) (Figure 1 and 2). Each of these 7 segments was scored: $0=$ no effacement, 1 = some effacement, 2 = complete effacement. The total score could range from 0 (no effacement) to 14 (complete effacement). Scores were given by two separate assessors and results averaged.

Craniectomy size was measured using a 3-dimensional reconstruction of the cranium and the volume measurement tool. If the difference in volume measured by the two assessors was greater than $3 \mathrm{~cm}^{2}$, a third assessor recorded an additional measurement and the two closest were retained and averaged.

Extent of subtemporal decompression was measured as the height of the remaining bone from the middle fossa floor to the inferior limit of the craniectomy, typically the lip of squamous temporal bone (Figure 3). It was taken at a point that correlated with the centre of the midbrain using a localizer. This equated 
to a point approximately $2 \mathrm{~cm}$ anterior to the root of the zygoma. The lower the figure the greater the middle fossa floor.

All data was stored in accordance with General Data Protection Regulations and the Data Protection Act 2018. It was kept in encrypted pseudo-anonymised files on a computer in a locked room at the hospital. Access was limited to the clinical research team. Data was analysed with simple statistics, two tailed student t-test, and simple linear regression with Bonferroni correction as appropriate.

\section{Results}

36 patients underwent a primary decompressive craniectomy for an acute subdural haematoma in a 5year period between June 2008 and August 2013 and fulfilled our strict inclusion and exclusion criteria.

The mean age was $36.1+/-12.5$ (range 16-62) and 69.4\% where male (25/36). The mechanism of injury was due to falls, road traffic accidents or assaults in $94.4 \%$ of cases (Table 1). The median GCS at presentation was 5 , with $76.2 \%$ presenting as severe traumatic brain injury (TBI) with a GCS of 3-8, $14.3 \%$ with moderate (GCS 9-13), and 9.5\% with mild TBI (GCS 14-15). Pre-operative pupil response was normal in $46.2 \%$ of cases, unilaterally fixed in $38.4 \%$ and bilaterally fixed and dilated in $15.4 \%$. The time taken between admission and operation was a median of 4.2 hours (interquartile range 1.8 hours 1 day).

There were an equal number of left and right sided convexity haematomas (18 of each). Two patients had an associated smaller extradural, and one patient had bilateral subdural haematomas (the larger left sided subdural was causing the mass effect requiring unilateral craniectomy and hence inclusion in this study). The median size of the haematoma was $8.3 \mathrm{~mm}$, with a midline shift of $8.4 \mathrm{~mm}$ and brainstem shift of $1.65 \mathrm{~mm}$ (Table 2). $63.9 \%$ of patients had contusions on imaging, and $88.9 \%$ had evidence of traumatic SAH blood.

The median craniectomy size was $88.7 \mathrm{~cm}^{2}$ (range $43.5-123.2 \mathrm{~cm}^{2}$ ) (Figure 4) and the median subtemporal decompression was $15.0 \mathrm{~mm}$ (range $3.0-57.2 \mathrm{~mm}$ ). There was no association with either the size of craniectomy or subtemporal decompression with the change in midline shift, brainstem shift or cisternal effacement score (Table 3). This can be seen graphically in Figures 5-7. In 17 patients (47.2\%) there was an element of brain herniation through the craniectomy defect on post-operative imaging, as reported by a consultant Neuroradiologist. There was no relationship between brain herniation through the craniectomy defect and pre-operative midline shift $(\mathrm{p}=0.679)$, brainstem shift $(\mathrm{p}=0.642)$, or craniectomy size $(\mathrm{p}=0.595)$ on a two-tailed t-test.

33 patients $(91.7 \%)$ had a post prognostication Magnetic Resonance Imaging (MRI) scan postoperatively. 20 (60.6\%) had no evidence of DAI on imaging, and $6(18.2 \%), 5(15.2 \%)$, and $2(6.1 \%)$ had evidence of grade 1,2 , and 3 DAI respectively. 
Long term follow-up was available in $97.2 \%$ of cases (35/36) with a median of 23.5 months (range 2.2 -109.6 months). There was no mortality in this cohort. The majority of the patients made a good recovery with $82.8 \%$ having a favourable modified Rankin score of 2 or less (Table 4). Additionally, there was no association between craniectomy size or subtemporal decompression and outcome $\left(\mathrm{R}^{2}=0.0092\right.$ and 0.0487 respectively). $100 \%$ of patients had a cranioplasty at a median of 9.3 months (range $1.6-53.1$ months).

\section{Discussion}

This study demonstrates a statistically significant reduction in post-operative midline shift, brainstem shift and cisternal effacement following primary craniectomy for acute subdural haematoma evacuation. However, the size of craniectomy or extent of subtemporal decompression showed a striking lack of correlation with the degree of midline shift, brainstem shift or cisternal effacement resolution. In this study, the size of the craniectomy and subtemporal decompression was patient and surgeon specific, with the aim to resolve midline shift and brainstem compression, and improve patient outcomes. This gave us the opportunity to pragmatically correlate different sizes of craniectomy with subtypes of brain shift.

Despite being one of the oldest and most common cranial neurosurgical procedures the exact indications and rationale for a primary craniectomy are still being elucidated. A large craniectomy with a subtemporal decompression is not a benign procedure and should only be undertaken if there is likely to be a benefit to the patient. Evidence suggests that primary decompressive craniectomy is performed in a significant proportion of acute subdural haematoma evacuations in the UK.[20] The decision to perform a craniectomy must be balanced against several negative consequences. There is the risk of surgical complication and morbidity, a cosmetic bony defect as well as risk of damage to the temporalis muscle/innervation, and the potential need for a later operation to insert a cranioplasty which itself carries additional risks including infection.[6, 14, 15, 24] It is important to know if the craniectomy size correlates with shift resolution, to determine if there is a critical size required, and to help decide if a craniotomy will suffice. The benefit of a primary craniectomy versus craniotomy for acute subdural haematomas is uncertain, and is being investigated in the RESCUE-ASDH trial.[19, 22]

The finding that craniectomy size does not correlate with the degree of midline shift can reasonably be explained by the assertion that the primary mediators of midline shift are a) residual haematoma and b) brain swelling and underlying parenchymal injury. The European Brain Injury Consortium had a mean craniectomy size of $67 \mathrm{~cm}^{2}$ and determined that less than $30 \mathrm{~cm}^{2}$ was inadequate.[8] By this definition, all the bone flaps in our study were of adequate size (Figure 4), which may explain the lack of correlation. Decompression is a secondary factor and any role it plays in resolution of midline shift did not show a correlation. It may be the case that more swollen brains, and worse injuries, received larger flaps, essentially masking any effect on reducing shift. Additionally, not all the cases were planned as primary decompressive craniectomies, some were planned as craniotomies, but swelling and intraoperative 
appearance may have led to the decision to leave the bone out. The confounding role of a progression in pathological process in the intervening time between the preoperative and postoperative scans also cannot be discounted. In $47 \%$ of cases there was herniation of brain through the craniectomy defect on post-operative imaging, suggesting a degree of brain swelling post-operatively. This showed no association with pre-operative shift or craniectomy size.

Subtemporal decompression was described by Harvey Cushing as a method of controlling pressure resulting from brain tumours and traumatic blast injuries.[9, 10] It was initially used as an isolated method without the larger craniectomy seen with modern decompressions.[2, 13, 16] Today, a craniectomy including the subtemporal decompression is common practice. However, it has associated risks, including bleeding (particularly with skull base fractures), need for a preauricular incision, prolonged operative time, lateral brain herniation, poor cosmesis and the risk of damaging the frontalis branch of the facial nerve. It is important to determine whether subtemporal decompression improves brain shift or cisternal effacement before continuing to routinely perform this additional step.

The theory behind subtemporal decompression is that any pressure generated at the middle fossa floor, for example due to brain swelling or contusions, will be transferred medially to the brainstem in the form of uncal herniation if there is not a sufficiently low craniectomy to allow expansion of the mass laterally. Although an intuitively appealing theory this has never been empirically tested. The subtemporal region is not a true 'closed box' with only a medial opening. Although there are bone limits anteriorly (greater wing of sphenoid), laterally (squamous temporal bone), inferiorly (fossa floor) and posteriorly (petrous ridge), the fossa is largely open superiorly apart from an anterior portion of the temporal pole covered by the lesser wing of sphenoid. This means that pressure transfer can occur from the middle fossa floor superiorly to the cerebral hemisphere, and vice versa. Whether pressure shifts brain medially or superiorly from the middle fossa will depend on the pressure differential within these spaces. By 'opening the box' with a craniectomy above the middle temporal fossa it may be the case that this route of pressure equalization is adequate to lead to a resolution in brainstem shift and cisternal effacement. The contribution of the subtemporal portion of this decompression was not evident in this study and it may be that it contributes far less to resolution of pressure on the brainstem than classically taught. Another factor to consider is the pliability of the skin and soft tissue lateral to the middle fossa after closure, in comparison to the convexity. The extra bulk of temporalis and tension provided by proximity to bone attachments may make expansion here less likely to occur, particularly in the context of a large hemispheric decompression.

The outcomes over a median of 23.5 months were very positive. Despite significant TBI, with patients having a median presentation GCS of 5 (three quarters of the cohort presented as a severe TBI and half had at least one fixed pupil), there were no mortalities and the majority of the patients made a good recovery. Our inclusion criteria necessitated a post-operative CT scan, so patients who were too unstable for a post-operative scan, and may have gone on to die, were excluded. In addition, as over $85 \%$ of our cohort had good outcomes, we found no association between pre-operative compression or craniectomy 
size and outcome. This is likely to be a result of too few patients having poor outcomes for conclusions to be reliably drawn.

There are several limitations to this study. It was a retrospective, single centre study, with a relatively small sample size, reflecting out strict inclusion and exclusion criteria. We were unable to elucidate whether the craniectomy was planned preoperatively or intraoperatively. A decision may provisionally be made on the preoperative scan but the final decision is usually made after the state of the brain and the space available after clot evacuation has been visualised. We were unable to assess whether the bone opening was increased intraoperatively, for example, as a result of unexpected brain swelling, and postoperative pupil response documentation was poor. ICP data was unavailable, so we were unable to compare pre and post-operative data and compare it to craniectomy size, or stratify it with pathology. However, brain herniation through the defect acted as a crude surrogate. We hope to assess this in future work.

We would argue that knowledge of these factors was not necessary to determine a correlation between extent of craniectomy and shift. This was a pragmatic analysis and demonstrated the real-life correlation between craniectomy size and various subtypes of brain shift. The lack of a clear linear relationship, or even a correlation, between craniectomy size and markers of brain shift urges us to re-examine the importance of the bone opening in a craniectomy for evacuation of subdural haematomas.

\section{Conclusion}

This study suggests that there is no clear relationship between craniectomy size or extent of subtemporal decompression and resolution of brain shift or outcome. This includes midline shift at the level of the foramen of Monro, shift at the level of the midbrain and cisternal effacement. Other factors, including adequacy of haematoma evacuation, surgeon's prediction of subsequent brain swelling, and the heterogenous nature of TBI, are likely to be critical.[5] Further studies should focus on larger prospective series or randomised controlled studies to assess the relative efficacy of large craniectomies and the role of subtemporal decompression.

\section{Funding}

No funding was received for this research.

\section{Conflict of Interest}

All authors certify that they have no affiliations with or involvement in any organization or entity with any financial interest (such as honoraria; educational grants; participation in speakers' bureaus; membership, employment, consultancies, stock ownership, or other equity interest; and expert testimony or patent-licensing arrangements), or non-financial interest (such as personal or professional 
relationships, affiliations, knowledge or beliefs) in the subject matter or materials discussed in this manuscript.

\section{Ethical approval}

This study was registered locally with the Clinical Effectiveness Unit as a Service Evaluation prior to data collection. Data was fully anonymised so formal consent was not required.

\section{Acknowledgements}

We would like to thank Angelos Triantafyllidis and Vejay Vakharia who helped with data collection.

\section{Figures}

Figure 1

CT scan at level of perimesencephalic cistern demonstrating the 4 borders used to calculate cisternal effacement score.

Figure 2

CT scan at level of quadrigeminal cistern demonstrating the 3 borders used to calculate the cisternal effacement score.

\section{Figure 3}

CT with bone windows demonstrating a subtemporal decompression as measured in the coronal plane.

\section{Figure 4 \\ Histogram of craniectomy size}

\section{Figure 5}

Graph to show the change in midline shift in relation to craniectomy size

\section{Figure 6}

Graph to show the change in brainstem shift in relation to subtemporal decompression

\section{Figure 7}

Graph to show the change in cisternal effacement in relation to subtemporal decompression

\section{Figure 8}

CT brain showing pre and post-operative midline shift

\section{Tables}




\begin{tabular}{|lll|}
\hline Mechanism & $\begin{array}{l}\text { Patients } \\
(\mathbf{n})\end{array}$ & $\begin{array}{l}\text { Percentage } \\
(\boldsymbol{\%})\end{array}$ \\
\hline Fall from height & 10 & 25.0 \\
Assault & 9 & 27.8 \\
Pedestrian versus vehicle & 6 & 11.1 \\
Other road traffic accident & 4 & 16.7 \\
Fall from standing & 5 & 13.9 \\
Pre-eclampsia & 1 & 2.8 \\
Unknown mechanism & 1 & 2.8 \\
\hline
\end{tabular}

Table 1: The mechanism of injury for acute subdural haemorrhage.

\begin{tabular}{|llll|}
\hline Variable & Pre-operative & Post-operative & p value \\
& & & \\
\hline Haematoma size $(\mathrm{mm})$ & $8.3(3.1-32.3)$ & & \\
Septum pellucidum midline shift $(\mathrm{mm})$ & $8.4(0-18.8)$ & $0.45(-12.1-12.1)$ & $<.00001$ \\
Brainstem shift $(\mathrm{mm})$ & $1.65(0-5.1)$ & $0(-2.6-3)$ & $<.00001$ \\
Cistern effacement score & $6(0-14)$ & $0.5(0-12)$ & $<.00001$ \\
\hline
\end{tabular}

Table 2: Pre and post-operative imaging measurements, including haematoma size, septum pellucidum midline shift, brainstem shift and cistern effacement score. Results given as median with range in brackets. $P$ values calculated with two tailed t-test. All p values are statistically significant.

\begin{tabular}{|ll|}
\hline Association & $\mathbf{R}^{\mathbf{2}}$ \\
\hline Craniectomy size and: & 0.0002 \\
Midline shift & 0.0133 \\
Brainstem shift & 0.049 \\
Cisternal effacement score & \\
& \\
Subtemporal decompression and: & \\
Midline shift & 0.0218 \\
Brainstem shift & 0.0008 \\
Cisternal effacement score & 0.0001 \\
\hline
\end{tabular}

Table 3: Association of craniectomy size and subtemporal decompression with the change in midline shift, brain stem shift and cisternal effacement score. Correlation co-efficient $\left(R^{2}\right)$ calculated with linear regression.

\begin{tabular}{|l|l|}
\hline Modified Rankin Scale & $\begin{array}{l}\text { Patients } \\
\mathbf{n}(\%)\end{array}$ \\
\hline 6 - death & 0 \\
\hline $5-$ severe disability & 0 \\
\hline
\end{tabular}




\begin{tabular}{|l|l|}
\hline $4-$ moderate severe disability & $5(14.3)$ \\
\hline $3-$ moderate disability & $1(2.9)$ \\
\hline $2-$ slight disability & $4(11.4)$ \\
\hline $1-$ no significant disability & $9(25.7)$ \\
\hline $0-$ no symptoms & $16(45.7)$ \\
\hline
\end{tabular}

Table 4: Patient outcomes on the Modified Rankin Scale. Data is given as raw numbers and percent. One patient was lost to follow up.

\section{References}

1. Akbik OS, Starling R V., Gahramanov S, Zhu Y, Lewis J (2019) Mortality and Functional Outcome in Surgically Evacuated Acute Subdural Hematoma in Elderly Patients. World Neurosurg 126:e1235-e1241

2. Alexander E, Ball MR, Laster DW (1987) Subtemporal decompression: Radiological observations and Current surgical experience. Br J Neurosurg 1(4):427-433

3. Artru F, Jourdan C, Convert J, Deschamps J, Guichard P, Chiara Y, Terrier A, Deleuze R, Duquesnel J (1989) An effacement score for basal cisterns to predict ICP level and outcome after closed head injury. Intracranial Press VII 29(4):273-279

4. Balestreri M, Czosnyka M, Hutchinson P, Steiner LA, Hiler M, Smielewski P, Pickard JD (2006) Impact of intracranial pressure and cerebral perfusion pressure on severe disability and mortality after head injury. Neurocrit Care 4(1):8-13

5. Bor-Seng-Shu E, De-Lima-Oliveira M, Nogueira RC, Almeida KJ, Paschoal EHA, Paschoal FM (2019) Decompressive craniectomy for traumatic brain injury: Postoperative TCD cerebral hemodynamic evaluation. Front Neurol. doi: 10.3389/fneur.2019.00354

6. Cabraja M, Klein M, Lehmann TN (2009) Long-term results following titanium cranioplasty of large skull defects. Neurosurg Focus 26(6):1-7

7. Chiewvit P, Tritakarn S, Nanta-aree S, Suthipongchai S (2010) Degree of midline shift from CT scan predicted outcome in patients with head injuries. J Med Assoc Thai 93(1):99-107

8. Compagnone C, Murray GD, Teasdale GM, Maas AIR, Esposito D, Princi P, D'Avella D, Servadei F (2005) The management of patients with intradural post-traumatic mass lesions: A multicenter survey of current approaches to surgical management in 729 patients coordinated by the European Brain Injury Consortium. Neurosurgery 57(6):1183-1191

9. Cushing H (1905) The establishment of cerebral hernia as a decompressive measure for inaccessible brain tumors : with the description of intermuscular methods of making the bone defect in temporal and occipital. Surgery, Gynecol Obstet 1(October):106-123

10. CUSHING H (1908) SUBTEMPORAL DECOMPRESSIVE OPERATIONS FOR THE INTRACRANIAL COMPLICATIONS ASSOCIATED WITH BURSTING FRACTURES OF THE SKULL. Ann Surg 47(5):641-644

11. Fearnside MR, Cook RJ, Mcdougall P, Mcneil RJ (1993) The Westmead head injury project outcome in severe head injury. A comparative analysis of pre-hospital, clinical and CT 
variables. Br J Neurosurg 7(3):267-279

12. Fleminger S, Ponsford J (2005) Long term outcome after traumatic brain injury. Br Med J 331(7530):1419-1420

13. Gower DJ, Lee KS, McWhorter JM, Pitts LH, Geisler FH (1988) Role of subtemporal decompression in severe closed head injury. Neurosurgery 23(4):417-422

14. Hill CS, Luoma AMV, Wilson SR, Kitchen N (2012) Titanium cranioplasty and the prediction of complications. Br J Neurosurg 26(6):832-837

15. Honeybul S, Ho KM (2014) Decompressive craniectomy for severe traumatic brain injury: The relationship between surgical complications and the prediction of an unfavourable outcome. Injury 45(9):1332-1339

16. Huang X, Wen L (2010) Technical considerations in decompressive craniectomy in the treatment of traumatic brain injury. Int J Med Sci 7(6):385-390

17. Jennett B, MacMillan R (1981) Epidemiology of head injury. Br Med J (Clin Res Ed) 282(6258):101-104

18. Kay A, Teasdale G (2001) Head injury in the United Kingdom. World J Surg 25(9):1210-1220

19. Kolias AG, Li LM, Guilfoyle MR, Timofeev I, Corteen EA, Pickard JD, Kirkpatrick PJ, Menon DK, Hutchinson PJ (2013) Decompressive craniectomy for acute subdural hematomas: Time for a randomized trial. Acta Neurochir (Wien) 155(1):187-188

20. Kolias AG, Scotton WJ, Belli A, et al (2013) Surgical management of acute subdural haematomas: Current practice patterns in the United Kingdom and the Republic of Ireland. Br J Neurosurg 27(3):330-333

21. Moussa WMM, Khedr WM, Elwany AH (2018) Prognostic significance of hematoma thickness to midline shift ratio in patients with acute intracranial subdural hematoma: a retrospective study. Neurosurg Rev 41(2):483-488

22. Phan K, Moore JM, Griessenauer C, Dmytriw AA, Scherman DB, Sheik-Ali S, Adeeb N, Ogilvy CS, Thomas A, Rosenfeld J V. (2017) Craniotomy Versus Decompressive Craniectomy for Acute Subdural Hematoma: Systematic Review and Meta-Analysis. World Neurosurg 101:677-685.e2

23. Quattrocchi KB, Prasad P, Willits NH, Wagner FC (1991) Quantification of midline shift as a predictor of poor outcome following head injury. Surg Neurol 35(3):183-8

24. Reid Gooch M, Gin GE, Kenning TJ, German JW (2009) Complications of cranioplasty following decompressive craniectomy: Analysis of 62 cases. Neurosurg Focus 26(6):1-7

25. Teasdale GM (1995) Head injury. J Neurol Neurosurg Psychiatry 58(5):526-539

26. Vibbert M, Mayer SA (2010) Early decompressive hemicraniectomy following malignant ischemic stroke: The crucial role of timing. Curr Neurol Neurosci Rep 10(1):1-3

27. Yılmaz İ, Ertem DH, Kılıç M, Altaş K, Mirhasilova M, Özdemir B, Tanrıverdi O, Yılmaz A (2019) Factors associated with mortality in acute subdural hematoma: Is decompressive craniectomy effective? Ulus Travma ve Acil Cerrahi Derg 25(2):147-153

28. Zumkeller M, Behrmann R, Heissler HE, Dietz H (1996) Computed tomographic criteria and survival rate for patients with acute subdural hematoma. Neurosurgery 39(4):708-713 
1

2

3

4

7

8

10

11

12

13

14

15

16

17

18

19

20

21

22

23

24

25

26

27

28

29

30

31

32

33

34

35

36

37

38

39

40

41

42

43

44

45

46

47

48

49

50

51

52

53

54

55

56

57

58

59

60

61

62

63

64

65 